\title{
Listed Waste History at Hanford Facility TSD Units
}

\author{
A. G. Miskho
}

Date Published

June 1996

Prepared for the U.S. Department of Energy Office of Environmental Restoration and Waste Management

\footnotetext{
(2) Westinghouse P.O. Box 1970

Hanford Company Richland, Washington
}

Henford Operations and Engineering Contractor for the

U.S. Department of Energy under Contract DE-AC06-87RL10930 
TRADLMARK DISCLAIMER

Reference herein to any opecific commercial product, process, or service by trade name, manufacturer, or otherwise, does not necessarily constitute or imply its endorsement, recommendation, or favoring by the United States Govermment or any agency thereof or its contractors or subcontractors.

This report has been reproduced from the best available copy. Available in paper copy and microfiche.

Available to the U.S. Department of Energy and its contractors from Office of Scientific and Technical Information P.O. Box 62

Oak Ridge, TN 37831

(423) $576-8401$

Available to the public from the U.S. Department of Commerce National Technical Information Service

5285 Port Royal Road

Springfield, VA 22161

(703) $487-4650$

Privied is the United Statce of America

DHSCLM-5.CHP (1-9) 


\section{RELEASE AUTHORIZATION}

Document

Number:

Document

Title:
WhC-EP-0857-1

Statement of Work for Analytical Sexvices Provided to Westinghouse Hanford Company by the Pacific Northwest National Laboratory Analytical Chemistry Laboratory

Release Date: $05 / 24 / 96$

This document was reviewed following the procedures described in WHC-CM-3-4 and is:

APPROVED FOR PUBLIC RELEASE

WhC Information Release Administration specialist:

V. 2

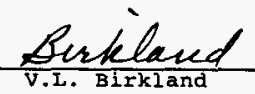

$6 / 14 / 96$ 



\section{LISTED WASTE HISTORY AT HANFORD FACILITY TSD UNITS}

Prepared by:

A. G. Miskho

Westinghouse Hanford Company

$5 / 21 / 56$

Date

Senior Engineer, Environmental Services

Approved by:

R. J. Landon,

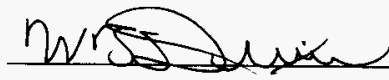

W. T. Dixon

West inghouse Hanford Company

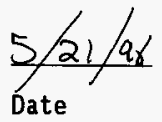

Director, Environmental Services

Bechtel Hanford, Inc.

Manager, Regulatory Support

Approved by:

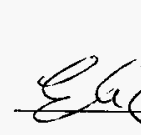

E. A. Flores

Pacific Northwest National Laboratories

Laboratory Environmental Compliance Manager

$5121 / 96$

$5 / 21 / 96$

Date

Date 
WHC-MR-0517 Rev. 0

This page intentionally left blank. 
This document was prepared to close out an occurrence report that Westinghouse Hanford Company issued on December 29, 1994. Occurrence Report RL-WHC-GENERAL-1994-0020 was issued because knowledge became available that could have impacted start up of a Hanford Site facility. The knowledge pertained to how certain wastes on the Hanford site were treated, stored, or disposed of. This document consolidates the research performed by Westinghouse Hanford Company regarding listed waste management at onsite laboratories that transfer waste to the Double-Shell Tank System.

Liquid and solid (non-liquid) dangerous wastes and mixed wastes at the Hanford site are generated from various site operations. These wastes may be sampled and characterized at onsite laboratories to meet waste management requirements. In some cases, the wastes that are generated in the field or in the laboratory from the analysis of samples require further management on the Hanford Site and are aggregated together in centralized tank storage facilities. The process knowledge presented herein documents the basis for designation and management of 242-A Evaporator Process Condensate, a waste stream derived from the treatment of the centralized tank storage facility waste (the Double-She17 Tank System). This document will not be updated as clean up of the Hanford site progresses. 
WHC-MR-0517 Rev. 0

This page intentionally left blank. 


\section{CONTENTS}

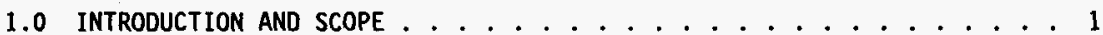

2.0 PURPOSE .............................. 2

3.0 PRESENTATION OF INFORMATION . . . . . . . . . . . . . . . . . 2

4.0 DESCRIPTIONS OF COLUMNS IN TABLE $1 . \ldots \ldots$ 4.1 TREATMENT, STORAGE, AND DISPOSAL UNIT NAME [UNIT CODES] . . . . . 4 4.2 LISTED WASTE CODES ON PART A FORM $3 . . . . . . . . . . . . . . .44$ 4.3 LISTED WASTE CODES MANAGED IN UNIT ..................... 4 4.4 TABLE 2 PG\#.......................... 4 $4.5 \mathrm{CO} O \mathrm{OP}$........................... . . . 4 4.6 AREA . . . . . . . . . . . . . . . 4 4.7 DOE/RL-88-21 MANUAL SECTION ................. 5

5.0 REFERENCES ........................... 7

\section{TABLES}

1. Treatment, Storage, and Disposal Units with $F, P, K$, or $U$ Waste Codes on Part A Form 3...................... . . T1-1

2. Listed Waste Management History at Treatment, Storage, and Disposai

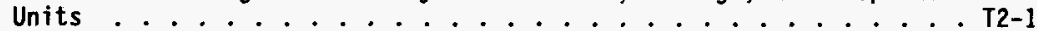




\section{ACRONYMS}

$\begin{array}{ll}\text { ASE } & \text { Area Solvent Evaporator } \\ \text { BHI } & \text { Bechtel Hanford Incorporated } \\ \text { CWC } & \text { Central Waste Complex } \\ \text { CY } & \text { calendar year } \\ \text { DST } & \text { double-shell tank } \\ \text { ECology } & \text { Washington State Department of Ecology } \\ \text { EDL } & \text { Engineering Development Laboratory } \\ \text { EII } & \text { Environmental Investigations Instruction } \\ \text { EPA } & \text { U.S. Environmental Protection Agency } \\ \text { GEA } & \text { gamma energy analysis } \\ \text { HEDL } & \text { Hanford Environmental Development Laboratory } \\ \text { HEHF } & \text { Hanford Environmental Health Foundation } \\ \text { HWTU } & \text { Hazardous Waste Treatment Unit } \\ \text { ICP } & \text { inductively coupled plasma } \\ \text { LDR } & \text { Land Disposal Restrictions } \\ \text { LERF } & \text { Liquid Effluent Retention Facility } \\ \text { LWDF } & \text { Liquid Waste Disposal Facility } \\ \text { NCAW } & \text { Neutralized Current Acid Waste } \\ \text { NRDWSF } & \text { Nonradioactive Dangerous Waste Storage Facility } \\ \text { Part A Form } & \text { Part A Form } 3 \text { Permit Application } \\ \text { PNNL } & \text { Pacific Northwest National Laboratory } \\ \text { PUREX } & \text { Plutonium Uranium Extraction } \\ \text { RCRA } & \text { Resource Conservation and Recovery Act of 1976 } \\ \text { REDOX } & \text { Reduction Oxidation (Facility) } \\ \text { RL } & \text { U.S. Department of Energy, Rich1and Operations office } \\ \text { RLWS } & \text { Radioactive Liquid Waste System } \\ \text { SAL } & \text { Shielded Analytical Laboratory } \\ \text { SST } & \text { single-shell tank } \\ \text { TCLP } & \text { Toxicity Characteristic Leaching Procedure } \\ \text { Tri-Party } & \text { Hanford Federal Facility Agreement and Consent Order } \\ \text { Agreement } & \text { transuranic } \\ \text { TRU } & \text { Treatment Storage, and Disposal } \\ \text { TSD } & \text { U.S. Testing, Inc. } \\ \text { UST } & \text { uitraviolet } \\ \text { UV } & \text { Washington Administrative Code } \\ \text { WAC } & \text { Waste Acid Treatment System } \\ \text { WATS } & \text { Westinghouse Hanford Company } \\ \text { WHC } & \text { Waste Receiving and Packaging } \\ \text { WRAP } & \\ \text { WSCF } & \\ & \text { Haste Sampling and Characterization Facility } \\ & \end{array}$




\section{LISTED WASTE HISTORY AT HANFORD FACILITY TSD UNITS}

\subsection{INTRODUCTION AND SCOPE}

This document contains a history of the management of listed wastes at Hanford Facility Treatment, Storage, and Disposal (TSD) units. Part of this history includes how Hanford Site laboratories support the characterization of wastes being managed at Hanford Facility TSD units. Some Hanford Site laboratories generate waste that is transferred to the Double-Shell Tank System, a TSD unit, for subsequent storage until treatment is made available for that waste. Samples of wastes sent to the Hanford Site laboratories are managed under the sample exclusion while they are being tested to determine their characteristics or composition. The sample exclusion is found in Washington Administrative Code (WAC), Chapter 173-303, "Dangerous Waste Regulations" in Section -071(3)(1). Once the sample exclusion has expired, all knowledge pertaining to the original sample becomes attached to the laboratory waste by virtue of the mixture and/or derived from rules in the Resource Conservation and Recovery Act of 1976 (RCRA).

There are more than 60 TSD units on the Hanford Site that must be either permitted or closed in accordance with the schedule identified in the Hanford Facility RCRA Permit. The Hanford Facility RCRA Permit defines most of the Hanford site as a facility and the TSD units as discrete locations on the Hanford Site where TSD can occur or has occurred. These locations are referred to as the "active portions" of the Hanford Facility as defined in WAC-173-303. These activities are outlined for the different TSD units or groups of TSD units in the latest revision of DOE/RL-88-21, Hanford Facility Dangerous Waste Part A Permit Application (DOE 1988). The Hanford Facility's Part A Permit Application consists of two different forms called a Form 1 and a Form 3. In many cases, a single TSD unit is contained in a Part A Form 3. There are situations, however, where a group of ISD units appear on a single Part A Form 3. The Hanford Federal Facility Agreement and Consent Order (Tri-Party Agreement) (Ecology et al. 1996e) Action Plan, Section 3.2, allows TSD units to be grouped to meet permitting needs. These forms are filed with the Washington State Department of Ecology (Ecology).

Listed waste managed at non-TSD unit locations on the Hanford Facility are not discussed in this document. During the cleanup of the Hanford Facility, it is expected that new listed waste sources will be discovered at non-TSD unit locations since historical information about how chemicals were used on the Hanford Facility must be applied to the management of remediated wastes (57 FR 37284).

This document does not discuss history of wastes that were designated for either the characteristics or criteria in WAC 173-303-090 and -100, respectively. For information on which of these waste codes are associated with TSD units, refer to the most current version of the TSD units' Part A Form 3. 


\subsection{PURPOSE}

The information contained in this document may be used by RCRA permitting organizations to maintain the Part A Form 3 of each TSD unit or group of units on the Hanford Facility when wastes are transferred between TSD units for subsequent treatment and disposal. It also can be used by program personnel to identify possible contained-in determinations, treatment equivalency demonstrations, and/or treatability variances for environmental media or hazardous debris. Much of the environmental media and hazardous debris at the Hanford Site is managed as a mixed waste as a result of contact or potential contact with listed wastes described in Table 1 and Table 2 of this document. This document can also be used as a reference for past decisions regarding listed waste. This information may be valuable in the preparation of Part $B$ Permit Applications or Closure/Post Closure permit Applications and/or Closure Plans.

\subsection{PRESENTATION OF INFORMATION}

Two tables are presented in this document. For a reader to obtain information about what listed wastes were managed in a TSD group/unit, Table I should be consulted first. Once the TSD group/unit has been identified, the reader can obtain detailed information about listed waste management from Table 2. Table 1 of this document identifies all TSD units that have a history of listed waste management, regardless if the TSD unit has been clean closed. This TSD unit list is a subset of all of the TSD units contained in DOE/RL-88-21. Because of the complexity of information at certain TSD units, a separate supplemental Table 2 has been prepared.

Table 2 of this document provides detailed information to supplement the information contained in Table 1. There are three categories of information in Table 2: Unit History, Samples, and Current. The Unit History information section describes the type of TSD units on the Part A Form 3 and whether any other Hanford Facility TSD units have managed the same waste. It also discusses any relevant waste designation aspects. The Samples information section indicates when listed wastes were sampled, and in some cases, the laboratory to which the samples were sent. The Current information describes a variety of subjects appropriate for the TSD unit.

of the more than 60 Part A Form 3 's identified in DOE/RL-88-21, a subset of those (42) appear in the tables contained in this document. The information is presented in the same way as it is presented in DOE/RL-88-2l, by geographical area on the Hanford Site. Information appearing in Tables 1 and 2 contain the following entries, in the order it appears below:

100 AREAS

183-H SOLAR EVAPORATION BASIMS

1301-N LIQUID WASTE DISPOSAL FACILITY

1325-N LIQUID WASTE DISPOSAL FACILITY 


\section{AREAS}

204-AR WASTE UNLOADING STATION

PUREX PLANT

HANFORD UASTE VITRIFICATION PLANT

200 AREA EFFLUENT TREATMENT FACILITY

200 WEST ASHPIT DEMOLITION SITE

218-E-8 BORROU PIT DEMOLITION SITE

242-A EVAPORATOR

GROUT TREATMENT FACILITY

T PLANT COAPLEX

B PLANT COAPLEX

222-S LABORATORY COMPLEX

2727-s STORAEE FACILITY

LIOUID EFFLUENT RETENTION FACILITY

DOUBLE-SHELL. TANK SYSTEM

HEXONE STORAGE AND TREATMENT FACILITY

224-T TRAHSURAMIC WASTE STORAGE AND ASSAY FACILITY

CENTRAL WASTE COMPLEX

WASTE RECEIVIHG AND PROCESSING

SINGLE-SHELL TANK SYSTEM

207-A SOUTH RETENTION BASIN

LOH-LEVEL BURIAL GROUNDS

216-A-37-1 CRIB

216-B-3 EXPANSION POWD

246-A-29 DITCH

216-8-3 MAIN POND

\section{AREA}

THERMAL TREATMENT TEST FACILITIES

300 AREA SOLVENT EVAPORATOR

300 AREA WASTE ACID TREATMENT SYSTEN

325 HAZARDOUS WASTE TREATMENT UNITS

BIOLOGICAL TREATMENT TEST FACILITIES

PHYSICAL \& CHEMICAL TREATMENT TEST FACILITIES

303-K STORAGE UNIT

305-B STORAGE FACILITY

332 STORAGE FACILITY

300 AREA PROCESS TRENCHES

600 AREA

HANFORD PATROL ACADENY DEMOLITION SITES

616 NONRADIOACTIVE DANGEROUS WASTE STORAGE FACILITY

600 AREA PURGEWATER STORAGE AMD TREATMENT FACILITY

NONRADIOACTIVE OANGEROUS WASTE LANDFILL

\subsection{DESCRIPTIONS OF COLUMNS IN TABLE 1}

This section provides a description of the columns in Table 1 of this document.

\subsection{TREATMENT, STORAGE, AND DISPOSAL UNIT NAME [UNIT CODES]}

The Treatment, Storage, and Disposal (TSD) unit name identified in this column is exactly as it appears in DOE/RL-88-21, Hanford Facility Dangerous Waste Part A Permit Application, Revision 13 (DOE 1988). The unit code in parentheses indicates the type of TSD unit that is permitted to operate. TSD units operating under interim status are deemed to have a permit to operate when a current Part $A$ form 3 is maintained in this document. The unit codes include the following: 


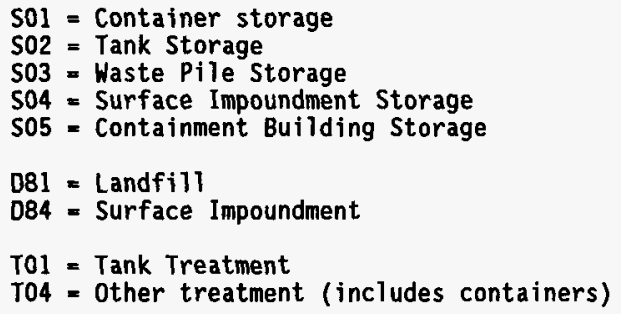

\subsection{LISTED WASTE CODES ON PART A FORM 3}

The listed waste codes identified in this column were obtained from reviewing the Part A Form 3, Permit Applications (Part A Form 3) contained in DOE/RL-88-21, Revision 13. The listed waste codes include any federal RCRA waste codes identified by a three-digit number preceded with an ' $F$, ' ' $K$,' ' $U$,' or ' $p$ ' letter. Ecology-regulated, state-only waste codes and Federalcharacteristic waste codes are purposely excluded from this document.

\subsection{LISTED MASTE CODES MANAGED IN UNIT}

The information presented in this column was developed by (1) reviewing all Form 5, "Annual Dangerous and Mixed Waste" TSD reports that were submitted to Ecology (Calendar Year [CY] 1984 to Present), (2) reviewing existing Ecology-approved RCRA Closure Plans and drafts within Westinghouse Hanford Company (WHC) and Bechtel Hanford Incorporated (BHI) for TSD units that are closing, (3) investigation of TSD unit operating records and generator files, (4) Tri-Party Agreement (Ecology et al. 1996e) unit manager or project manager meeting minutes, and (5) discussions with personnel who are knowledgeable of historical TSD unit operations. The accuracy of the information in this column is critical for properly developing the next two columns.

\subsection{TABLE 2 PG\#}

The information in this column identifies the page number on which information appears in Table 2, a supplemental table containing the listed waste management history at the TSD group/unit.

\section{$4.5 \mathrm{CO}-\mathrm{OP}$}

The information in this column identifies the Hanford Facility major contractor identified as the Co-operator on the Part A Form 3.

\subsection{AREA}

Information in this column identifies the Hanford Facility geographical area(s) where the TSD group/unit is located. 


\subsection{DOE/RL-88-21 MANUAL SECTION}

This information identifies the section number where the Part $A$ Form 3 appears in DOE/RL-88-21 and the associated Part A Form 3 revision number in DOE/RL-88-21, Revision 13. 
WHC-MR-0517 Rev. 0

This page intentionally left blank. 
Table 1. Treatment, Storage, and Disposal Units with F, P, K, or U Waste Codes on Part A Form 3. (Page 1 of 6 )

\begin{tabular}{|c|c|c|c|c|c|c|}
\hline TSD UNIT NAME [UNIT CODES] & $\begin{array}{l}\text { LISTED WASTE CODES ON PART A } \\
\text { FORM } 3\end{array}$ & $\begin{array}{c}\text { LISTED WASTE COOES MANAGED IN } \\
\text { UNIT }\end{array}$ & TABLE 2 & CO-OP & AREA & $\begin{array}{c}\text { DOE/AL-88-21 } \\
\text { MANUAL SECTION : }\end{array}$ \\
\hline $\begin{array}{l}\text { 183-H SOLAR EVAPORATION } \\
\text { BASINS [TO1, SO2] }\end{array}$ & U123, P030, P120, P029, P106, & $\begin{array}{c}\text { U123-Formic acid } \\
\text { P030-Cyanides (soluble sal ts } \\
\text { and complexes) } \\
\text { p029-Copper Cyenide } \\
\text { P106-Sodium Cyenide } \\
\text { P098-Potass i Lm Cyanide } \\
\text { P120-Vanadium Pentoxide }\end{array}$ & $1-2$ & BHI & $100 \mathrm{H}$ & $\begin{array}{l}4.1 .1 .4 \\
\text { Rev. } 4\end{array}$ \\
\hline $\begin{array}{l}\text { 1301-N LIQUID WASTE DISPOSAL } \\
\text { FACILITY [D81] }\end{array}$ & F003, U133 & U133-Hydrazine & 3 & BHI & 100* & $\begin{array}{l}4.1 .2 .1 \\
\text { Rev. } 5\end{array}$ \\
\hline $\begin{array}{l}1325-N \text { LIOUID WASTE DISPOSAL } \\
\text { FACILITY [DB1] }\end{array}$ & F003, U133 & $\begin{array}{l}\text { U133-Hydrazine } \\
\text { F003-Methanol }\end{array}$ & 4 & BHI & 100N & $\begin{array}{l}4.1 .2 .2 \\
\text { Rev. } 5\end{array}$ \\
\hline $\begin{array}{l}\text { 204-AR WASTE UNLOAD ING } \\
\text { STATION [TO4] } \\
\end{array}$ & F001-F005, F039 & $\begin{array}{c}\text { Same as Double-Shell Tank } \\
\text { System identified in this table }\end{array}$ & 5 & WHC & $200 E$ & $\begin{array}{c}4.2 .1 .10 \\
\text { Rev. } 3 \\
\end{array}$ \\
\hline $\begin{array}{l}\text { PUREX PLANT } \\
\text { [T01, S02, S05] }\end{array}$ & $\begin{array}{c}\text { NONE. Not applicable besed } \\
\text { on Tri-Party Agreement PUREX } \\
\text { Plant unit managers meeting } \\
\text { minutes dated } \\
\text { August } 9,1995 .\end{array}$ & $\begin{array}{c}\text { Fo03- acetone, n-butyl alcohol, } \\
\text { xylene } \\
\text { F005- toluene }\end{array}$ & $\overline{5}$ & WHC & $200 E$ & $\begin{array}{l}4.2 .1 .11 \\
\text { Rev. } 6\end{array}$ \\
\hline $\begin{array}{l}\text { HANFORD WASTE VITRIFICATION } \\
\text { PLANT }[T O 1, \mathrm{TO4}, \mathrm{SO2,} \text { SO5, } \\
\text { SO1] }\end{array}$ & F003, F005 & MONE & 6 & WHC & $200 E$ & $\begin{array}{l}4.2 .1 .12 \\
\text { Rev. } 4\end{array}$ \\
\hline $\begin{array}{l}200 \text { AREA EFFLUENT TREATMENT } \\
\text { FACILITY [TO1，S02, SO1] }\end{array}$ & F001-F005, F039 & F001-F005 & 6 & WHC & $200 E$ & $\begin{array}{l}4.2 .1 .13 \\
\text { Rev. } 1\end{array}$ \\
\hline $\begin{array}{l}200 \text { WEST ASHPIT DEMOLITION } \\
\text { SITE [T04] }\end{array}$ & $\begin{array}{l}\text { P003, U019, U056, U098, U108, } \\
\text { U112, U117, U133, U135, U154, } \\
\text { U213, U220 }\end{array}$ & 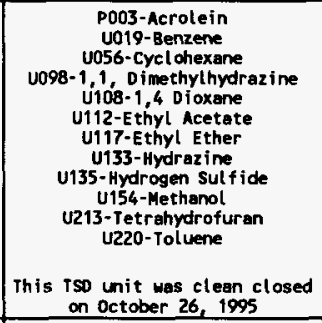 & 7 & WHC & $200 \mathrm{w}$ & $\begin{array}{l}4.2 .1 .2 \\
\text { Rev. } 4\end{array}$ \\
\hline $\begin{array}{l}\text { 218-E-8 BORROW PIT DEMOLITION } \\
\text { SITE [TO4] }\end{array}$ & U108, U160 & $\begin{array}{c}\text { U108-1,4 Dioxane } \\
\text { U160-Methyl Ethyl Ketone } \\
\text { Hydroxide } \\
\text { This TSD unit was clean closed } \\
\text { on October } 26,1995\end{array}$ & 7 & WHC & $200 E$ & $\begin{array}{l}4.2 .1 .3 \\
\text { Rev. } 4\end{array}$ \\
\hline
\end{tabular}


Table 1. Treatment, Storage, and Disposal Units with $F, P, K$, or $U$ Waste Codes on Part $A$ Form 3. (Page 2 of 6 )

\begin{tabular}{|c|c|c|c|c|c|c|}
\hline TSO UNIT NAME [UNIT CODES] & $\begin{array}{c}\text { LISTED WASTE CODES ON PART A } \\
\text { FORM } 3 \\
\end{array}$ & $\begin{array}{l}\text { LISTED WASTE CODES MANAGED IN } \\
\text { UNIT }\end{array}$ & $\begin{array}{c}\text { TABLE 2 } \\
\text { Pg: } \\
\end{array}$ & CO-OP & AREA & $\begin{array}{l}\text { DOE/RL-88-21 } \\
\text { MANUAL SECTION }\end{array}$ \\
\hline 242-A EVAPORATOR [TO4] & F001-F005，F039 & $\begin{array}{c}\text { Same as Double-shell Tank } \\
\text { system identified in this table }\end{array}$ & 8 & WHC & $200 E$ & $\begin{array}{l}4.2 .1 .4 \\
\text { Rev. } 5\end{array}$ \\
\hline $\begin{array}{l}\text { GROUT TREATMENT FACILITY } \\
\text { [TO4, T02, 502, T01, DB1] }\end{array}$ & $F 001, F 002, F 003, F 005$ & MONE & 8 & WHC & $200 E$ & $\begin{array}{l}4.2 .1 .5 \\
\text { Rev. } 4\end{array}$ \\
\hline $\begin{array}{l}\text { T PLANT COMPLEX [S02, 505, } \\
\text { T01, T04, S01, T041 }\end{array}$ & $\begin{array}{c}\text { S02, T01, T04, S05: F001- } \\
\text { F005, F039 } \\
\text { S01, r04: F001-F005, F020-23, } \\
\text { F026-28, F039, MAYY U ANO P } \\
\text { HASTES }\end{array}$ & $\begin{array}{l}\text { S02, T01, T04, S05: Same as } \\
\text { Double-sheli Tank System } \\
\text { identified in this table } \\
\text { 501, T04: Many }\end{array}$ & 9 & HHC & 2000 & $\begin{array}{l}4.2 .1 .6 \\
\text { Rev. } 5\end{array}$ \\
\hline $\begin{array}{l}\text { 8 PLANT COMPLEX [S02, T01, } \\
\text { S01. S05] }\end{array}$ & $\begin{array}{l}\text { NCAN TANKS S02, T01: } \\
\text { F001-F005 } \\
\text { LLW TANKS S02, TO1: F001-F005 } \\
\text { ORGANIC WASTE TANKS S02: } \\
\text { F001-F005 } \\
\text { LLW CONC'N TO1: } \\
\text { F001-F005 } \\
\text { CONTAIMMENT BUILDING S05: } \\
\text { FO01-F005 }\end{array}$ & $\begin{array}{c}\text { All units: Same as oouble- } \\
\text { Shell Tank System identified in } \\
\text { this table } \\
\text { Units will never manage F039 } \\
\text { wastes }\end{array}$ & 10 & NHC & $200 E$ & $\begin{array}{l}4.2 .1 .8 \\
\text { Rev. } 3\end{array}$ \\
\hline $\begin{array}{l}\text { 222-5 LABORATORY COMPLEX } \\
\text { is02, 101, S011 }\end{array}$ & $\begin{array}{l}\text { S02, T01: F001-F005, F039 } \\
\text { s01: } F 001=F 005, F 027, \text { F039 }\end{array}$ & \begin{tabular}{|c|} 
S02, T01: Same as Double-Shell \\
Tank System identified in this \\
teble \\
S01: F001- \\
1,1, Trichloroethane \\
F002- \\
Methyl lene Chl or ide \\
F003- Acetone, Xyl ene \\
F005- Methyl ethyl Ketone, \\
Tol uene \\
F027-Pentachlorophenol \\
\end{tabular} & $11-13$ & UHC & $200 \mathrm{w}$ & $\begin{array}{l}4.2 .1 .9 \\
\text { Rev. } 3\end{array}$ \\
\hline 2727-S STORAGE FACILITY [S01] & F001-F005, FO27, MAMY U AMD P & \begin{tabular}{|c|} 
Many \\
This iso unit was clean closed \\
on June 27, 1995
\end{tabular} & 13 & WHC & $200 \mathrm{~W}$ & $\begin{array}{l}4.2 .2 .1 \\
\text { Rev. } 2\end{array}$ \\
\hline $\begin{array}{l}\text { LIOUID EFFLUENT RETENTION } \\
\text { EACILITY [S04] (1) }\end{array}$ & F001-F005, F039 & \begin{tabular}{c|} 
Some as Double-Shell Tank \\
System identified in this table
\end{tabular} & 14 & Мнс & $200 E$ & $\begin{array}{l}4.2 .2 .10 \\
\text { Rev. } 3 \\
\end{array}$ \\
\hline
\end{tabular}


Table 1. Treatment, Storage, and Disposal Units with F, P, K, or $U$ Waste Codes on Part $A$ Form 3. (Page 3 of 6 )

\begin{tabular}{|c|c|c|c|c|c|c|}
\hline TSD UNIT NAME [UNIT CODES] & $\begin{array}{l}\text { UISTED WASTE CODES ON PART A } \\
\text { FORM } 3 \\
\end{array}$ & \begin{tabular}{|l} 
LISTED WASTE CODES MANAGED IN \\
UNIT \\
\end{tabular} & \begin{tabular}{|c|} 
TABLE 2 \\
$p g$ P $^{2}$ \\
\end{tabular} & CO-OP & AREA & $\begin{array}{c}\text { DOE/RL-88-21 } \\
\text { MANUAL SECTION }\end{array}$ \\
\hline $\begin{array}{l}\text { DOUBLE-SHELL IANK SYSTEM } \\
\text { [S02, T01] }\end{array}$ & F001-F005, F039 & $\begin{array}{c}\text { F001-1,1,1 Trichloroethane } \\
\text { F002-Methylene chloride } \\
\text { F003-Acetone, Hethyl Isobutyl } \\
\text { Ketone } \\
\text { F004-Cresols and cresylic acid } \\
\text { (-cresol and p-cresol) } \\
\text { F005-Methyl Ethyl Ketone }\end{array}$ & $15-16$ & UHC & $200 \mathrm{E}$ & $\begin{array}{l}4.2 .2 .2 \\
\text { Rev. } 7\end{array}$ \\
\hline $\begin{array}{l}\text { HEXOME STORAGE AND TREATMENT } \\
\text { FACILIIY [S02, } \mathrm{TO4}, \mathrm{S01]} \\
\end{array}$ & $F 003$ & $\begin{array}{c}\text { Fo03-Methyl Isobutyl Ketone } \\
\text { (Hexone) }\end{array}$ & 17 & BHI & $200 \mathrm{~W}$ & $\begin{array}{l}4.2 .2 .3 \\
\text { Rev. } 3 \\
\end{array}$ \\
\hline $\begin{array}{l}\text { 224-T IRANSURANIC MASTE } \\
\text { SIORAGE AND ASSAY FACILITY } \\
\text { [SO1] }\end{array}$ & $\begin{array}{l}\text { F001-F005, F020-23, F026-28, } \\
\text { MANY U AND P WASTES }\end{array}$ & $\begin{array}{c}\text { F001-F005: same as Double- } \\
\text { Shell Tank System identified in } \\
\text { this table } \\
\text { U123, P029, P030, P098, P106, } \\
\text { P120 }\end{array}$ & 17 & WHC & $200 \mathrm{w}$ & $\begin{array}{l}4.2 .2 .6 \\
\text { Rev. } 4\end{array}$ \\
\hline
\end{tabular}


Table 1. Treatment, Storage, and Disposal Units with F, P, K, or U Waste Codes on Part A Form 3. (Page 4 of 6 )

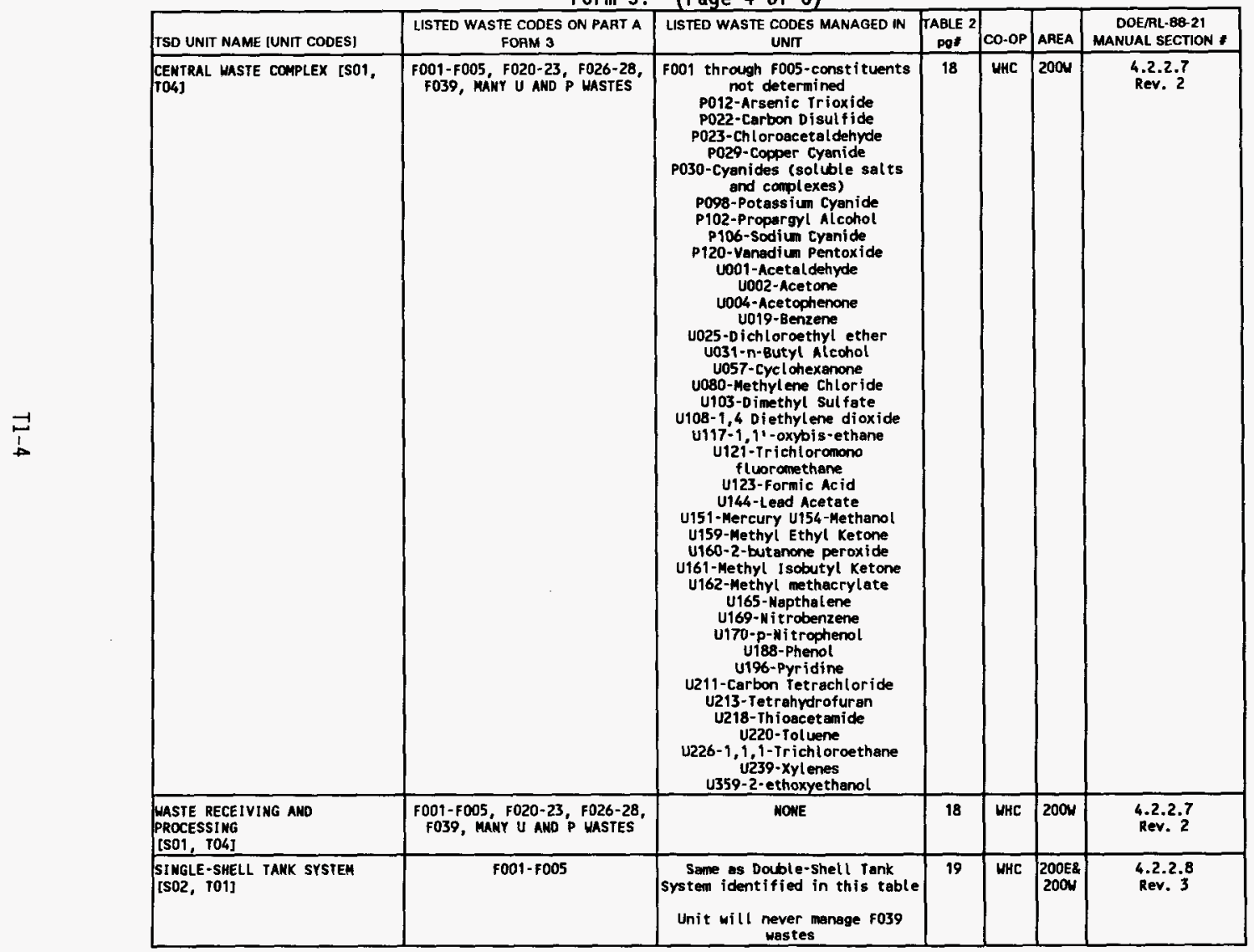


Table 1. Treatment, Storage, and Disposal Units with F, P, K, or $U$ Waste Codes on Part A Form 3. (Page 5 of 6 )

\begin{tabular}{|c|c|c|c|c|c|c|}
\hline TSD UNIT NAME (UNIT CODES) & \begin{tabular}{|c|}
$\begin{array}{l}\text { LIS TED WASTE CODES ON PART A } \\
\text { FORM } 3\end{array}$ \\
\end{tabular} & $\begin{array}{l}\text { LISTED WASTE CODES MANABED IN } \\
\text { UNIT }\end{array}$ & $\begin{array}{c}\text { TABLE } 2 \\
\text { PQ: }\end{array}$ & CO-OP & AREA & \begin{tabular}{|c|} 
DOE/RL-68-21 \\
MANUAL SECTION \\
\end{tabular} \\
\hline $\begin{array}{l}207-A \text { SOUTH RETENTION BASIN } \\
\text { [S04] }\end{array}$ & F001-F005 & $\begin{array}{c}\text { Seme as Double-Shell Tank } \\
\text { System identified in this table } \\
\text { Unit will never manage f039 } \\
\text { wastes }\end{array}$ & 19 & Whe & $200 E$ & $\begin{array}{l}4.2 .2 .9 \\
\text { Rev. } 1\end{array}$ \\
\hline $\begin{array}{l}\text { LOU-LEVEL BURIAL GROUNDS } \\
\text { [DB1] }\end{array}$ & $\begin{array}{c}\text { F001-F005, F028, F039, MANY U } \\
\text { AND P MASTES }\end{array}$ & Many & 20 & WHC & $\begin{array}{l}200 E 8 \\
200 \mathrm{~W} \\
\end{array}$ & $\begin{array}{l}4.2 .3 .1 \\
\text { Rev. } 7\end{array}$ \\
\hline 216-A-37-1 CRIB [D81] & F001-F005 & $\begin{array}{c}\text { Same as Double-Shell Tank } \\
\text { System identified in this table } \\
\text { Unit will never manage F039 } \\
\text { wastes }\end{array}$ & 20 & BHI & $200 E$ & $\begin{array}{l}4.2 .3 .10 \\
\text { Rev. } 2\end{array}$ \\
\hline $\begin{array}{l}\text { 216-8-3 EXPANSIOW PONO [T02, } \\
\text { P84] }\end{array}$ & 4133 & \begin{tabular}{|c|} 
U133-Hydrazine \\
This Tso unit was clean closed \\
on June 27, 1995
\end{tabular} & 21 & WHC & $200 E$ & $\begin{array}{l}4.2 .3 .11 \\
\text { Rev. } 0\end{array}$ \\
\hline 216-A-29 DITCH [T04, D84] & 4133 & U133-Hydrazine & 21 & BHI & $200 E$ & $\begin{array}{l}4.2 .3 .4 \\
\text { Rev. } 3\end{array}$ \\
\hline 216-B-3 MAIN POWD [T02, 084] & 4133 & U133-Hydrazine & 22 & BHI & $200 E$ & $\begin{array}{l}4.2 .3 .5 \\
\text { Rev. } 5\end{array}$ \\
\hline $\begin{array}{l}\text { THERMAL TREATMENT TEST } \\
\text { FACILITIES [TO4] }\end{array}$ & 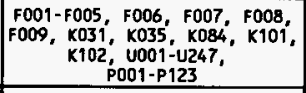 & MOME & 22 & PNML & 300 & $\begin{array}{l}4.3 .1 .10 \\
\text { Rev. } 0\end{array}$ \\
\hline $\begin{array}{l}300 \text { AREA SOLVEMT EVAPORATOR } \\
\text { [TO1, S01] }\end{array}$ & F001, F002, F003, F005 & \begin{tabular}{|c|} 
F001- Tetrachloroethyl ene, \\
Trichloroethylene, \\
Methylene chhloride \\
F002-1,1 Trichloroethane \\
F003. Ethyl acetate \\
F005- Methyl Ethyl Ketone \\
This TSD unit was clean closed \\
on June 27, 1995
\end{tabular} & 23 & WHC & 300 & $\begin{array}{l}4.3 .1 .4 \\
\text { Rev. } 4\end{array}$ \\
\hline $\begin{array}{l}300 \text { AREA WASTE ACID TREATMENT } \\
\text { SYSTEM [T01, S01, S02, T04] }\end{array}$ & $\begin{array}{c}300 \text { Area WATS-T01, S02, T04: } \\
\text { P120, U123 (1) } \\
311 \text { Tanks-T01, S01: NONE }\end{array}$ & MONE & $23-24$ & WHC & 300 & $\begin{array}{l}4.3 .1 .5 \\
\text { Rev. } 3\end{array}$ \\
\hline $\begin{array}{l}\text { 325 HAZARDOUS UASTE TREATMENT } \\
\text { UNI TS [S01, T04] }\end{array}$ & $\begin{array}{c}\text { F001-F005, F027, F039, K011, } \\
\text { K013, K048, K049, K050, K051, } \\
\text { K052, KANY U AND P WASTES }\end{array}$ & $\begin{array}{c}\text { Same as Double-shell rank } \\
\text { system identified in this table }\end{array}$ & $25-26$ & PMML & 300 & $\begin{array}{l}4.3 .1 .7 \\
\text { Rev. } 3\end{array}$ \\
\hline $\begin{array}{l}\text { BIOLOGICAL TREATMENT TEST } \\
\text { FACILITIES [TO4] } \\
\end{array}$ & $\begin{array}{c}\text { F001-F005, F027, } \\
\text { U001-U247, P001-P123 }\end{array}$ & NONE & 26 & PMML & 300 & $\begin{array}{l}4.3 .1 .8 \\
\text { Rev. } 0 \\
\end{array}$ \\
\hline $\begin{array}{l}\text { PHYSICAL \& CHEMICAL TREATMENT } \\
\text { TEST FACILIIIIS }[S 01, \text { T04] }\end{array}$ & $\begin{array}{l}\text { S01: F002, F003, F005 } \\
\text { T04: F001-F005, K048, K049, } \\
\text { K050, K051, K052, MANY U AND } \\
\text { P WASTES }\end{array}$ & $\begin{array}{c}\text { Same as Double-Shell Tank } \\
\text { System identified in this table } \\
\text { Unit will never manage } 0039 \\
\text { wastes }\end{array}$ & 26 & PMNL & 300 & $\begin{array}{l}4.3 .9 .9 \\
\text { Rev. } 1\end{array}$ \\
\hline
\end{tabular}


Table 1. Treatment, Storage, and Disposal Units with $F, P, K$, or $U$ Waste Codes on Part $A$ Form 3. (Page 6 of 6 )

\begin{tabular}{|c|c|c|c|c|c|c|}
\hline TSD UNIT NAME [UNIT CODES] & $\begin{array}{c}\text { LISTED WASTE COOES ON PART A } \\
\text { FORM } 3 \\
\end{array}$ & \begin{tabular}{|c} 
LISTED WASTE CODES MANAGED NN \\
UNIT
\end{tabular} & TABLE 2 & co-op & AREA & $\begin{array}{c}\text { DOE/RL-88-21 } \\
\text { MANUAL SECTION } \\
\end{array}$ \\
\hline 303-K STORAGE UHIT [S01] & F001, F002, F003, F005 & $\begin{array}{l}\text { F001 or F002- } \\
\text { Tetrachl oroethylene, } \\
1,1,1 \text { trichloroethars, } \\
\text { Irichloroethylene } \\
\text { F003- Ethyl acetate } \\
\text { F05- Methyl Ethyl } \\
\text { Ketone, Toluene }\end{array}$ & 27 & НHC & 300 & $\begin{array}{l}4.3 .2 .2 \\
\text { Rev. } 6\end{array}$ \\
\hline 305-B SIORAGE FACILITY [S01] & $\begin{array}{c}\text { F001-F005, F027, } \\
\text { P001-P123, U001-U249, U328, } \\
\text { U353, U359 }\end{array}$ & Many & 27 & PNNL & 300 & $\begin{array}{l}4.3 .2 .3 \\
\text { Rev. } 1\end{array}$ \\
\hline 332 STORAGE FACILITY [S01] & $\begin{array}{l}\text { F001-F005, F027, } \\
\text { MANY U AND'P WASTES }\end{array}$ & MONE & 27 & PNAL & 300 & $\begin{array}{l}4.3 .2 .4 \\
\text { Rev. } 0\end{array}$ \\
\hline $\begin{array}{l}300 \text { AREA PROCESS TRENCHES } \\
\text { [D84] }\end{array}$ & $\mathrm{F} 001, \mathrm{~F} 002, \mathrm{~F} 003, \mathrm{~F} 005, \mathrm{U} 210$ & $\begin{array}{l}\text { F001- } \\
\text { Tetrachloroethylene, } \\
\text { Trichloroethyl ene, } \\
\text { F002-Methyleme Chloride } \\
\end{array}$ & $28-29$ & WHC & 300 & $\begin{array}{l}4.3 .3 .1 \\
\text { Rev. } 4\end{array}$ \\
\hline $\begin{array}{l}\text { HAAFORD PATROL ACANEAY } \\
\text { DEMOLITION SITES [TTOG] }\end{array}$ & $\begin{array}{l}\text { P022, P048, P105, U108, U117, } \\
\text { U133, U213, U234 }\end{array}$ & $\begin{array}{l}\text { P022-Carbon Disulfide } \\
\text { P048- 2,4 Dinitrophenol } \\
\text { P105- Sodium Azide } \\
\text { U108-1,4 oioxane } \\
\text { U117-Ethyl Ether } \\
\text { U133-Hydrazine } \\
\text { U213-Tetrahydrofuran } \\
\text { U234- } 1,3,5-\text { Irinitrobenzene } \\
\text { This isD unit was clean closed } \\
\text { on October } 26,1995\end{array}$ & 29 & WHC & 600 & $\begin{array}{l}4.5 .1 .1 \\
\text { Rev. } 4\end{array}$ \\
\hline $\begin{array}{l}\text { 616 NONRADIOACTIVE DANGEROUS } \\
\text { HASTE STORAGE FACILITY [S01] }\end{array}$ & $\begin{array}{l}\text { FO01-F012, F019-F028, } \\
\text { MANY U AND P KASTES }\end{array}$ & Many & 30 & UHC & 600 & $\begin{array}{l}4.5 .2 .1 \\
\text { Rev. } 5 \\
\end{array}$ \\
\hline $\begin{array}{l}\text { 600 AREA PURGEUATER STORAGE } \\
\text { AND TREATMENT FACILITY [T04, } \\
\text { SO5] }\end{array}$ & F001-F003 & MOME & 30 & UHC & 600 & $\begin{array}{l}4.5 .2 .2 \\
\text { Rev. } 1\end{array}$ \\
\hline $\begin{array}{l}\text { WONRADIOACTIVE DANGEROUS } \\
\text { MASTE LANDFILL [DB1] }\end{array}$ & $\begin{array}{c}\text { F001-F005, } \\
\text { MANY U WASTES, PO10, P012, } \\
\text { P022, P030, P048, P096, P098, } \\
\text { P106 }\end{array}$ & Many & 31 & BHI & 600 & $\begin{array}{l}4.5 .3 .1 \\
\text { Rev. } 4\end{array}$ \\
\hline
\end{tabular}


Table 2. Listed Waste Management History at Treatment, Storage, and Disposal Units.

(Sheet 1 of 31 )

\begin{tabular}{|c|c|}
\hline TSD Unit & Listed Waste History \\
\hline $\begin{array}{l}\text { 183-H SOLAR } \\
\text { EVAPORATION } \\
\text { BASINS }\end{array}$ & $\begin{array}{l}\text { Unit history: The 183-H Solar Evaporation Basin \#1 received listed waste shipments from } \\
\text { the } 300 \text { Area Waste Acid Treatment System (WATS) via tanker truck in } 1976 \text { and } 1977 \text {. The } \\
\text { lifetime of the } 183-\mathrm{H} \text { Solar Evaporation Basins as a waste disposal unit was from } \\
\text { October } 1973 \text { to November } 1985 \text {. In addition to tanker truck discharges, certain wastes } \\
\text { were poured directly into Basin \#1 when safety issues surrounded the disposal of the } \\
\text { waste. Cyanide wastes meeting the discarded chemical product listing description were } \\
\text { poured directly into the basins and were not transported by tanker truck. All of this } \\
\text { information is documented in D0E/RL-88-04, Revision 3, 183-H Solar Evaporation Basins } \\
\text { Closure/Post-Closure Plan (DOE 1991), and is included in the Hanford Facility RCRA } \\
\text { Permit Dangerous Waste portion as Attachments } 11 \text { through 15. The listed waste from } \\
\text { Basin \#1 was pumped and mixed with waste in Basins \#2 through \#4 so that the waste in } \\
\text { all four basins were listed wastes. Thousands of drums containing solidified/stabilized } \\
\text { basin waste were shipped to the Central Waste Complex (CWC) for continued storage as } \\
\text { mixed waste. }\end{array}$ \\
\hline & $\begin{array}{l}\text { Samples: Waste - The waste in the 183-H Basins was sampled three different times } \\
\text { according to the } 183-H \text { Solar Evaporation Basin Closure/Post-Closure Plan (DOE 1991a). } \\
\text { The first sampling event (October 1984) resulted in samples being shipped to Pacific } \\
\text { Northwest National Laboratory (PNNL) for analysis. Analysis from this sampling event } \\
\text { was completed in three PNNL buildings: } 3720,3708 \text {, and the } 329 \text { Building. PNNL cannot } \\
\text { identify any knowledge about the disposition of analytical wastes that were generated } \\
\text { from analysis or any unused sample portions. The second sampling effort (January 1986) } \\
\text { involved samples that were sent to the } 222-S \text { Laboratory for selected radiological and } \\
\text { chemical analysis (including heavy metals). From this sampling effort, heavy metals } \\
\text { analysis was requested. } 222-S \text { Laboratory analytical instrumentation that analyzes heavy } \\
\text { metals, discharges the analytical wastes via a hard piped drain line to the } \\
222-5 \text { Laboratory Complex tank system so that the aqueous waste that was generated from } \\
\text { analysis was mixed in the laboratory waste water stream that was transferred to the } \\
\text { Double-Shell Tank (DST) System. The third sampling event (March 1987) involved analysis } \\
\text { of samples at an offsite laboratory, TMA/Norcal in Richmond, California. Waste sampling } \\
\text { activities continue in order to support information needed to dispose of the waste that } \\
\text { is stored in the CWC. Approximately 5,000 samples have been sent to the } \\
\text { Weston Laboratory, an offsite laboratory, for analysis from the } 377 \text { Building in the } \\
300 \text { Area. The } 377 \text { Building received drums of 183-H Solar Evaporation Basin waste (as a } \\
\text { sample) from the CWC. }\end{array}$ \\
\hline
\end{tabular}


Table 2. Listed Waste Management History at Treatment, Storage, and Disposal Units. (Sheet 2 of 31 )

\begin{tabular}{|c|c|}
\hline TSD Unit & Listed Waste History \\
\hline $\begin{array}{l}\text { 183-H SOLAR } \\
\text { EVAPORATION } \\
\text { BASINS (cont.) }\end{array}$ & 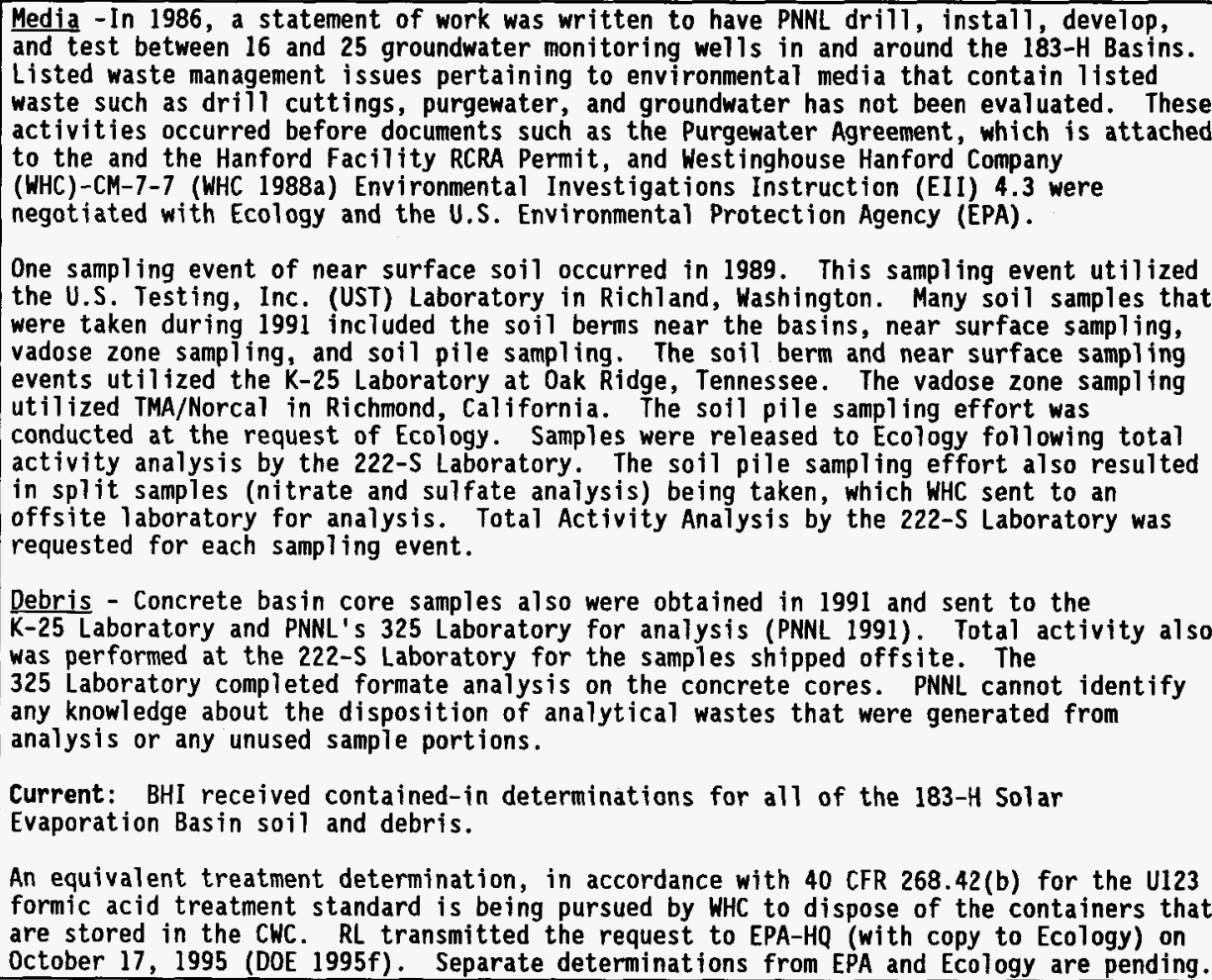 \\
\hline
\end{tabular}


Table 2. Listed Waste Management History at Treatment, Storage, and Disposal Units.

(Sheet 3 of 31)

\begin{tabular}{|c|c|}
\hline TSD Unit & Listed Waste History \\
\hline $\begin{array}{l}\text { 1301-N LIQUID } \\
\text { WASTE DISPOSAL } \\
\text { FAC IL ITY }\end{array}$ & 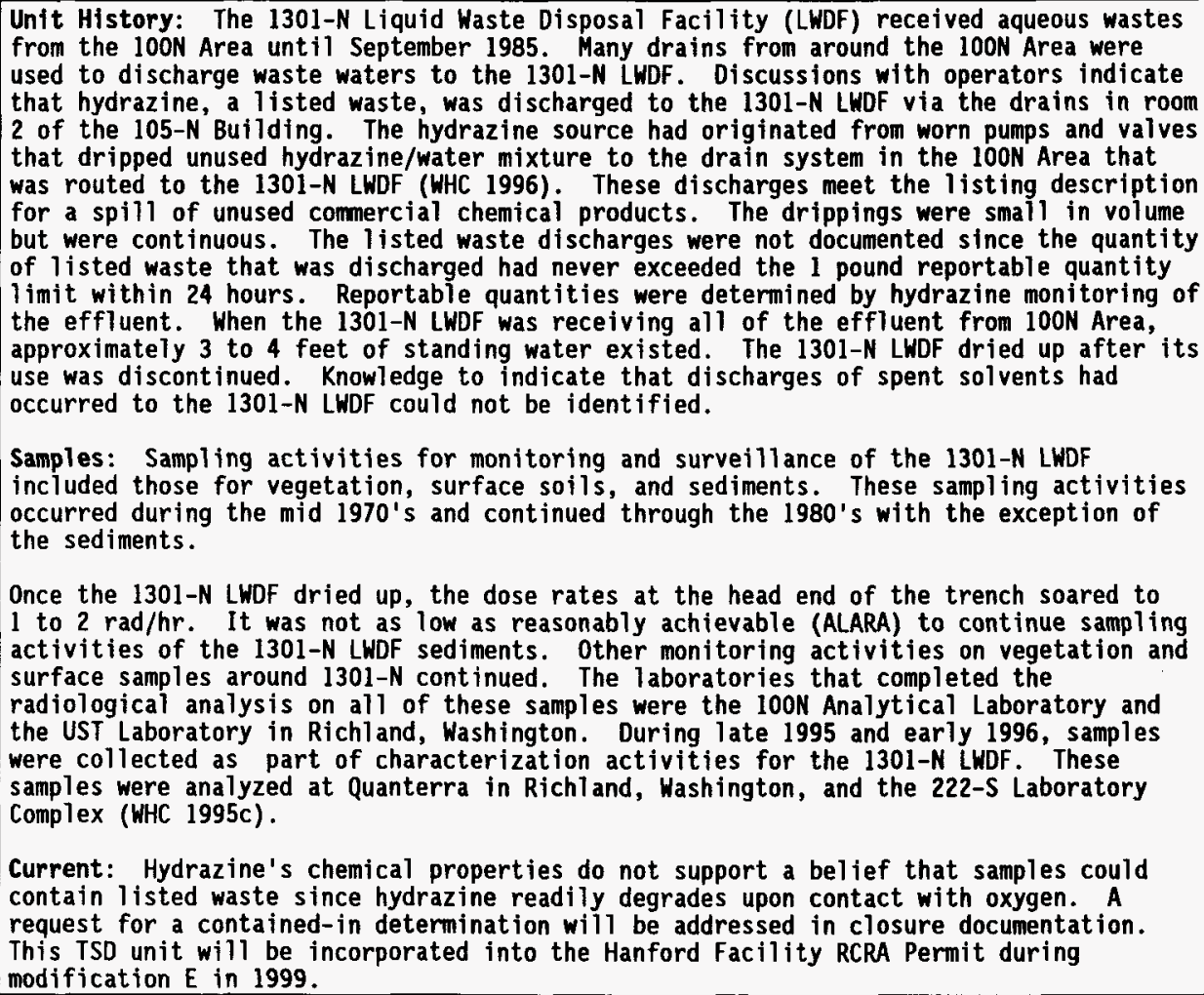 \\
\hline
\end{tabular}


Table 2. Listed Waste Management History at Treatment, Storage, and Disposal Units.

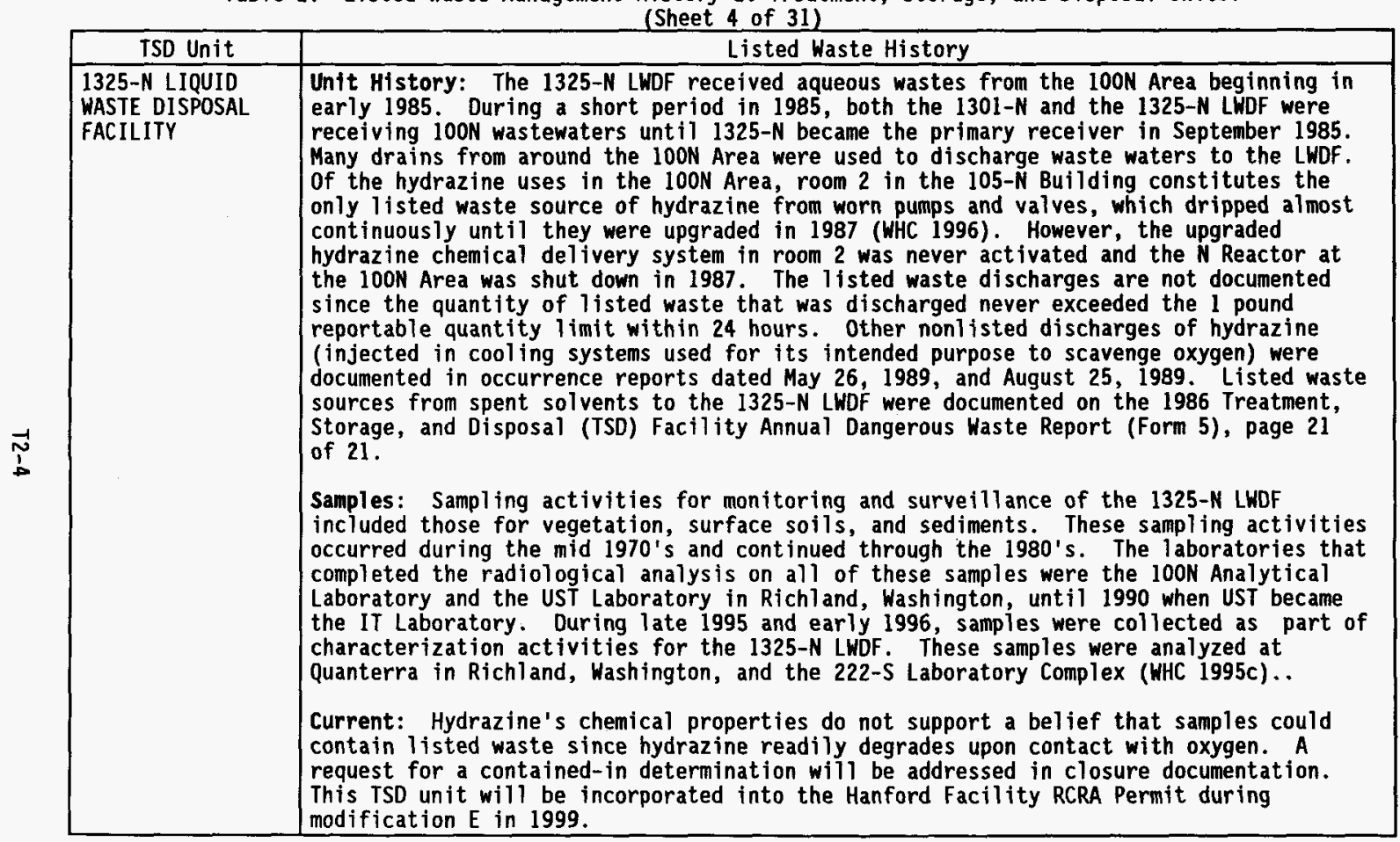


Table 2. Listed Waste Management History at Treatment, Storage, and Disposal Units. (Sheet 5 of 31 )

\begin{tabular}{|c|c|}
\hline TSD Unit & Listed Waste History \\
\hline $\begin{array}{l}\text { 204-AR WASTE } \\
\text { UNLOADING STATION }\end{array}$ & $\begin{array}{l}\text { Unit History: This unit accepts liquid wastes from Hanford Site sources for transfer } \\
\text { into the DST System. Wastes can be accepted either by tanker truck or by railcar into } \\
\text { this unit. Listed waste managed in this unit are dependent on the types of wastes } \\
\text { received from across the Hanford Site. The 204-AR Waste Unloading Station does not } \\
\text { generate listed wastes unique to Hanford Facility TSD units. Listed wastes receipts } \\
\text { have included those from the } 222-S \text { Laboratory Complex via tanker truck, the } 340 \text { Facility } \\
90 \text {-day tank system via railcar, and from the T-Plant Complex via railcar. } \\
\text { Samples: Sample evaluation is irrelevant since this unit does not generate its own } \\
\text { listed wastes. } \\
\text { Current: Since this unit receives listed wastes and nonlisted waste, all wastes managed } \\
\text { in this unit are considered listed wastes. The Part B Permit Application for this TSO } \\
\text { unit will be combined with the Part B Permit Application for the DST System during the } \\
\text { Hanford Facility RCRA Permit modification E in } 1999 \text {. }\end{array}$ \\
\hline PUREX PLANT & $\begin{array}{l}\text { Unit History: The PUREX Plant was constructed in } 1956 \text { and was used for the recovery of } \\
\text { uranium and plutonium from irradiated reactor fuel. The PUREX Plant is undergoing } \\
\text { deactivation, a transition phase in its closure during which past process solutions and } \\
\text { chemicals are being removed. Listed waste management in the PUREX Plant was associated } \\
\text { only with spent solvents from operations in the PUREX Plant laboratory where small } \\
\text { quantities of F-listed wastes were introduced into the PUREX Plant process. The } 1 \text { isted } \\
\text { constituents included acetone, xylene, n-butyl alcohol, and toluene. } \\
\text { Samples: The samples are not being investigated because of the } 1 \text { isted waste issue } \\
\text { resolution obtained through the Tri-Party Agreement (Ecology et al. 1996e) unit managers } \\
\text { meeting minutes. } \\
\text { Current: The listed waste issues at the PUREX plant were resolved on August 9, 1995, } \\
\text { when language was entered into the PUREX Plant Tri-Party Agreement (Ecology et al. } \\
1996 e) \text { unit managers meeting minutes concerning listed waste management (DOE et al. } \\
1995 \mathrm{~h} \text {. Attachment } 6 \text { of these meeting minutes contains the information pertaining to } \\
\text { listed waste management. The more important points can be paraphrased as: (1) the } \\
\text { listed waste codes will not be added to any Part A Form } 3 \text {, (2) waste managed or } \\
\text { generated from the PUREX Plant will not be designated as } 1 \text { isted waste, and (3) at the } \\
\text { time of final disposition of any waste from the PUREX Plant including the storage } \\
\text { tunnels, certain cribs, and vessels, all listed waste constituents must be addressed in } \\
\text { the sampling plan and in the assessment of final disposal options. }\end{array}$ \\
\hline
\end{tabular}


Table 2. Listed Waste Management History at Treatment, Storage, and Disposal Units.

(Sheet 6 of 31 )

\begin{tabular}{|c|c|}
\hline TSD Unit & Listed Waste History \\
\hline $\begin{array}{l}\text { HANFORD WASTE } \\
\text { VITRIFICATION } \\
\text { PLANT }\end{array}$ & $\begin{array}{l}\text { Unit History: Unit not constructed. This TSD unit may treat the high-level fraction of } \\
\text { DST System and Single-Shell Tank (SST) System wastes. } \\
\text { Samples: Not applicable. } \\
\text { Current: Not applicable. }\end{array}$ \\
\hline $\begin{array}{l}200 \text { AREA EFFLUENT } \\
\text { TREATMENT } \\
\text { FACILITY }\end{array}$ & $\begin{array}{l}\text { Unit History: This newly constructed TSD unit began operation in late } 1995 \text {. The TSD } \\
\text { unit processes listed wastes and delists those wastes. The delisting point in the TSD } \\
\text { unit is at the back end of the process in the verification tanks. Treatment residues } \\
\text { derived from listed wastes will be generated and sent to the CWC and/or the mixed waste } \\
\text { landfill in the Low-Level Burial Grounds. Wastes initially will be received for } \\
\text { processing from the Liquid Effluent Retention Facility (LERF) containing } \\
\text { 242-A Evaporator process condensate. }\end{array}$ \\
\hline & $\begin{array}{l}\text { Samples: Samples of } 200 \text { Area Effluent Treatment Facility wastes are sent to the Waste } \\
\text { Sampling and Characterization Facility (WSCF) Laboratory for analysis. }\end{array}$ \\
\hline
\end{tabular}


Table 2. Listed Waste Management History at Treatment, Storage, and Disposal Units. (Sheet 7 of 31 )

\begin{tabular}{|c|c|}
\hline TSD Unit & Listed Waste History \\
\hline $\begin{array}{l}200 \text { WEST ASHPIT } \\
\text { DEMOLITION SITE }\end{array}$ & $\begin{array}{l}\text { Unit History: This TSD unit managed listed waste in two detonation events, one in } \\
\text { November } 1984 \text { and the other in June } 1986 \text { (DOE 1994a). The discarded chemical products } \\
\text { ident ified on the unit's Part A Form } 3 \text { were detonated in the unit. This TSD unit is } \\
\text { included as a Part } V \text { unit in the Hanford Facility RCRA Permit Dangerous Waste portion as } \\
\text { Attachment } 21 \text {. } \\
\text { Samples: The only samples taken were soil samples acquired on July } 12 \text {, 1994, and a } \\
\text { second round of soil samples on December } 16 \text {, } 1994 \text {. Field screening was performed by the } \\
\text { Solid Waste Analysis Team on May } 12 \text {, } 1994 \text {. The July } 12 \text { samples were sent offsite for } \\
\text { analysis to the Knoxville, Tennessee, IT Laboratory through their local Richland, } \\
\text { Washington, affiliate. The second round of samples were analyzed by the new } \\
\text { Environmental Analytical Laboratory in the looN Area of the Hanford Facility. No } \\
\text { samples were taken following the two detonation events. } \\
\text { Current: All listed waste concerns were addressed through the Hanford Facility RCRA } \\
\text { Permit closure process. This is based on information pertaining to the efficiency by } \\
\text { which chemicals are destroyed through detonation and the time that has elapsed from the } \\
\text { last event at the TSD unit (DOE 1994a). The TSD unit has been clean closed as of } \\
\text { October } 25 \text {, 1995. }\end{array}$ \\
\hline $\begin{array}{l}\text { 218-E-8 BORROW } \\
\text { PIT DEMOL ITION } \\
\text { SITE }\end{array}$ & $\begin{array}{l}\text { Unit History: This TSD unit managed listed waste during one detonation event (DOE } \\
1994 d) \text {. The discarded chemical products identified on the Part A Form } 3 \text { were detonated } \\
\text { in November 1984. This TSD unit is a Part } V \text { unit included in the Hanford Facility RCRA } \\
\text { Permit Dangerous Waste portion as Attachment } 20 \text {. } \\
\text { Samples: The only protocol samples taken were soil samples acquired on July 12, } 1994 \text {. } \\
\text { Field screening was performed by the Solid Waste Analysis Team on May } 16,1994 \text {. The } \\
\text { July } 12 \text { samples were sent for analysis to the Knoxville, Tennessee, IT Laboratory } \\
\text { through their local Richland, Washington, affiliate. No samples were taken following } \\
\text { the detonation event. } \\
\text { Current: All listed waste concerns were addressed through the Hanford Facility RCRA } \\
\text { Permit closure process. This is based on DOE/RL-92-53, 218-E-8 Borrow Pit Demolition } \\
\text { Site Closure Plan (DOE 1992) information pertaining to the efficiency by which chemicals } \\
\text { are destroyed through detonation and the time that has elapsed from the last event at } \\
\text { the TSD unit. The TSD unit has been clean closed as of October } 25,1995 \text {. }\end{array}$ \\
\hline
\end{tabular}


Table 2. Listed Waste Management History at Treatment, Storage, and Disposal Units. (Sheet 8 of 31 )

\begin{tabular}{|c|c|}
\hline TSD Unit & Listed Waste History \\
\hline $\begin{array}{l}\text { GROUT TREATMENT } \\
\text { FACILITY }\end{array}$ & $\begin{array}{l}\text { Unit History: This TSD unit never managed listed waste since it did not treat } \\
\text { DST System waste. The campaign processed in the Grout Treatment Facility was } \\
\text { nondangerous phosphate-sulfate waste from the 100N Area. } \\
\text { Samples: Not applicable. } \\
\text { Current: Not applicable. }\end{array}$ \\
\hline
\end{tabular}


Table 2. Listed Waste Management History at Treatment, Storage, and Disposal Units.

(Sheet 9 of 31)

\begin{tabular}{|c|c|}
\hline TSD Unit & Listed Waste History \\
\hline T PLANT COMPLEX & 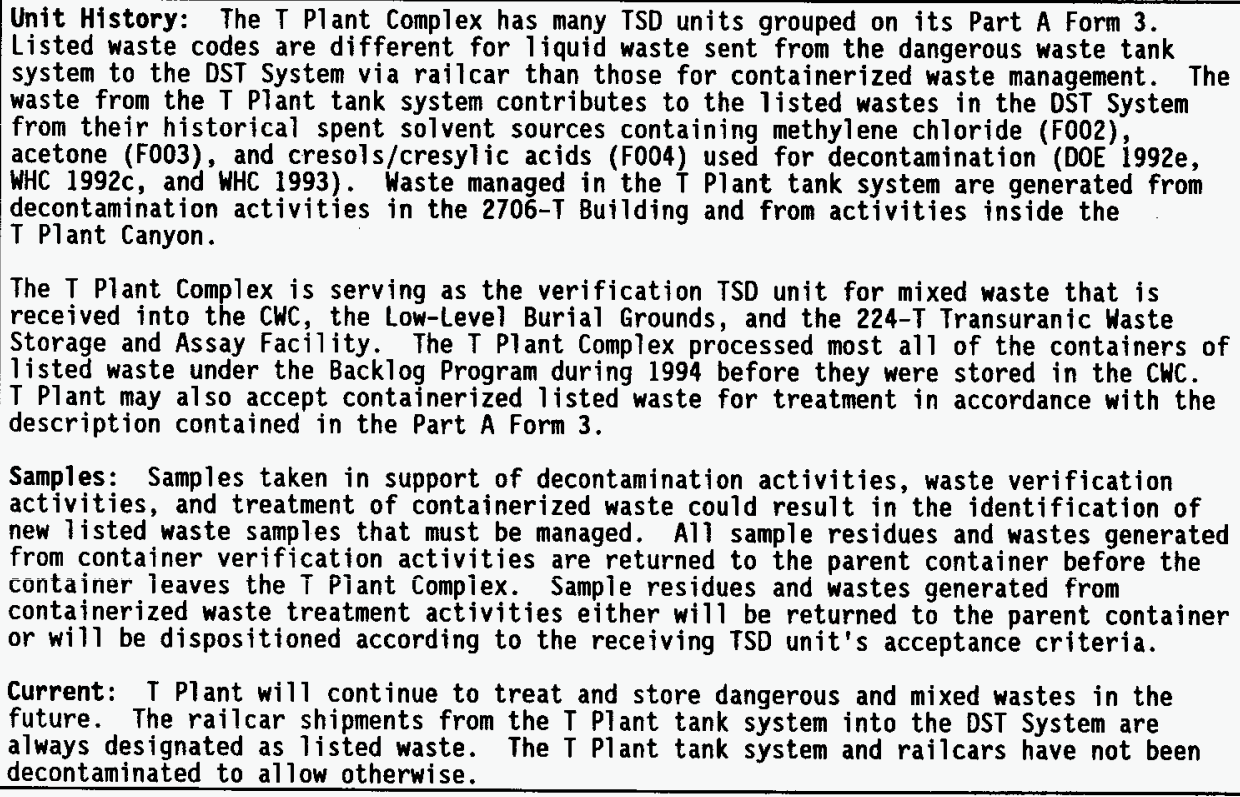 \\
\hline
\end{tabular}




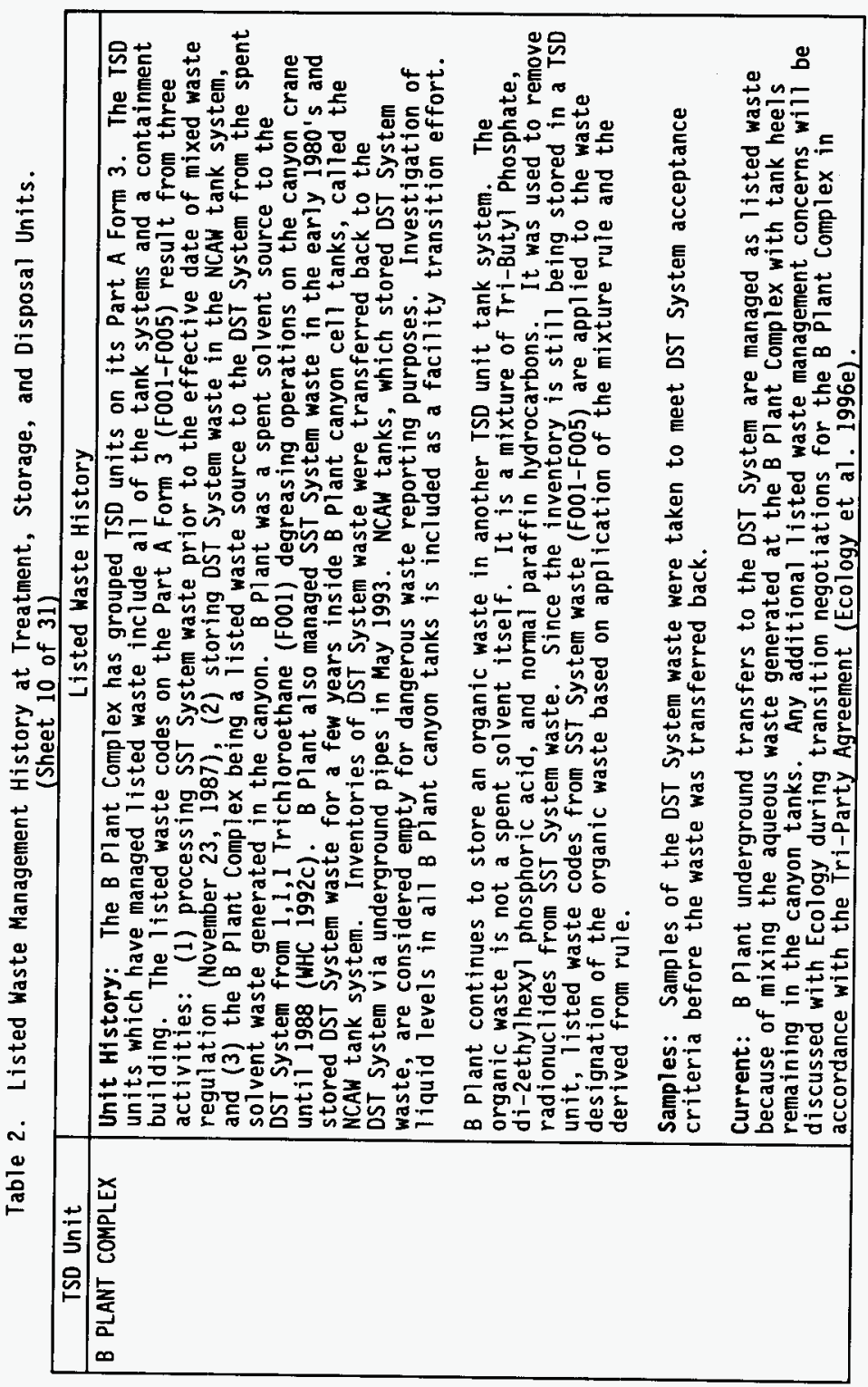


Table 2. Listed Waste Management History at Treatment, Storage, and Disposal Units. (Sheet 11 of 31)

\begin{tabular}{|c|c|}
\hline TSD Unit & Listed Waste History \\
\hline $\begin{array}{l}\text { 222-S LABORATORY } \\
\text { COMPLEX }\end{array}$ & 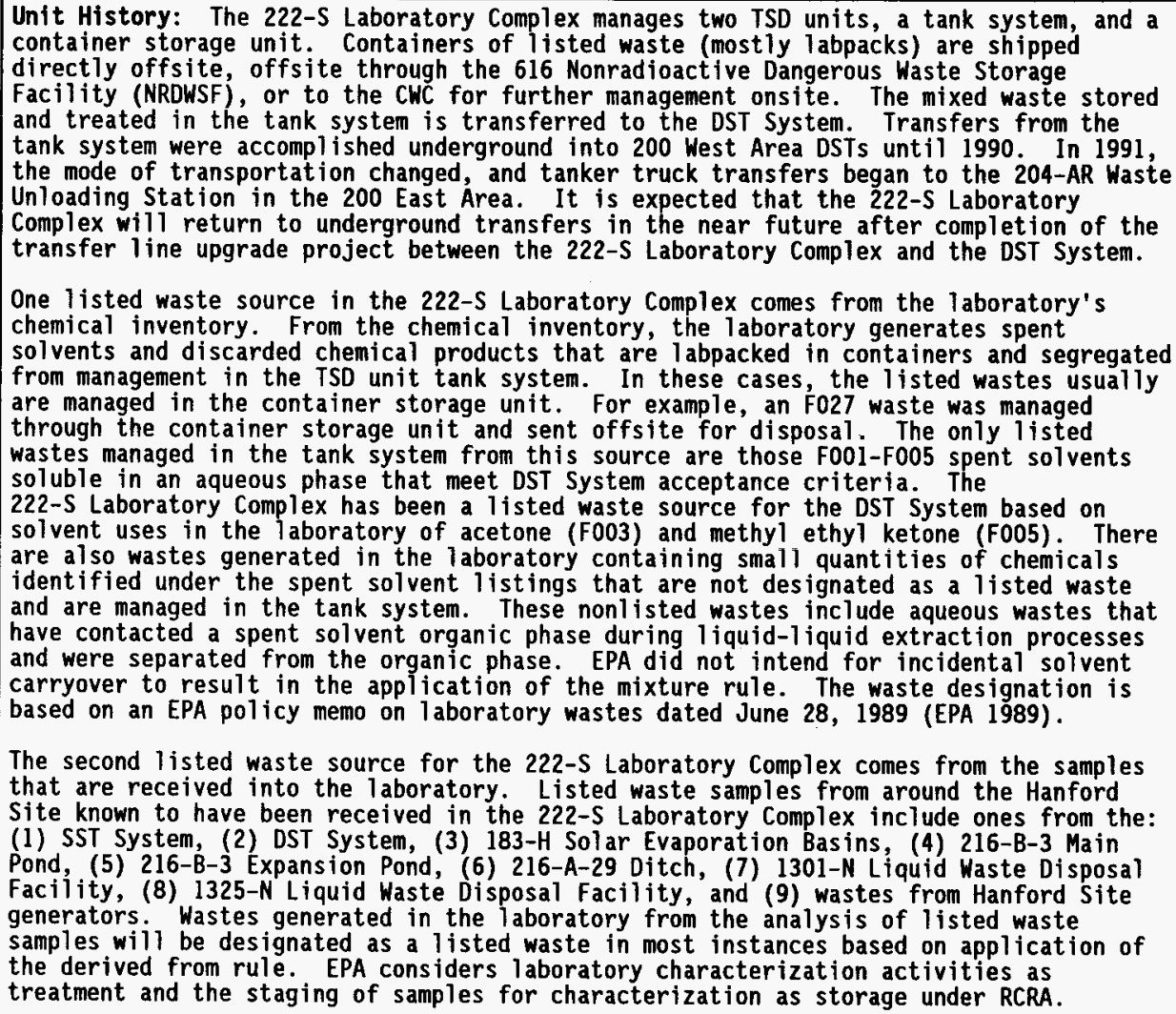 \\
\hline
\end{tabular}


Table 2. Listed Waste Management History at Treatment, Storage, and Disposal Units. (Sheet 12 of 31 )

\begin{tabular}{|c|c|}
\hline TSD Unit & Listed Waste History \\
\hline $\begin{array}{l}\text { 222-S LABORATORY } \\
\text { COMPLEX (cont.) }\end{array}$ & 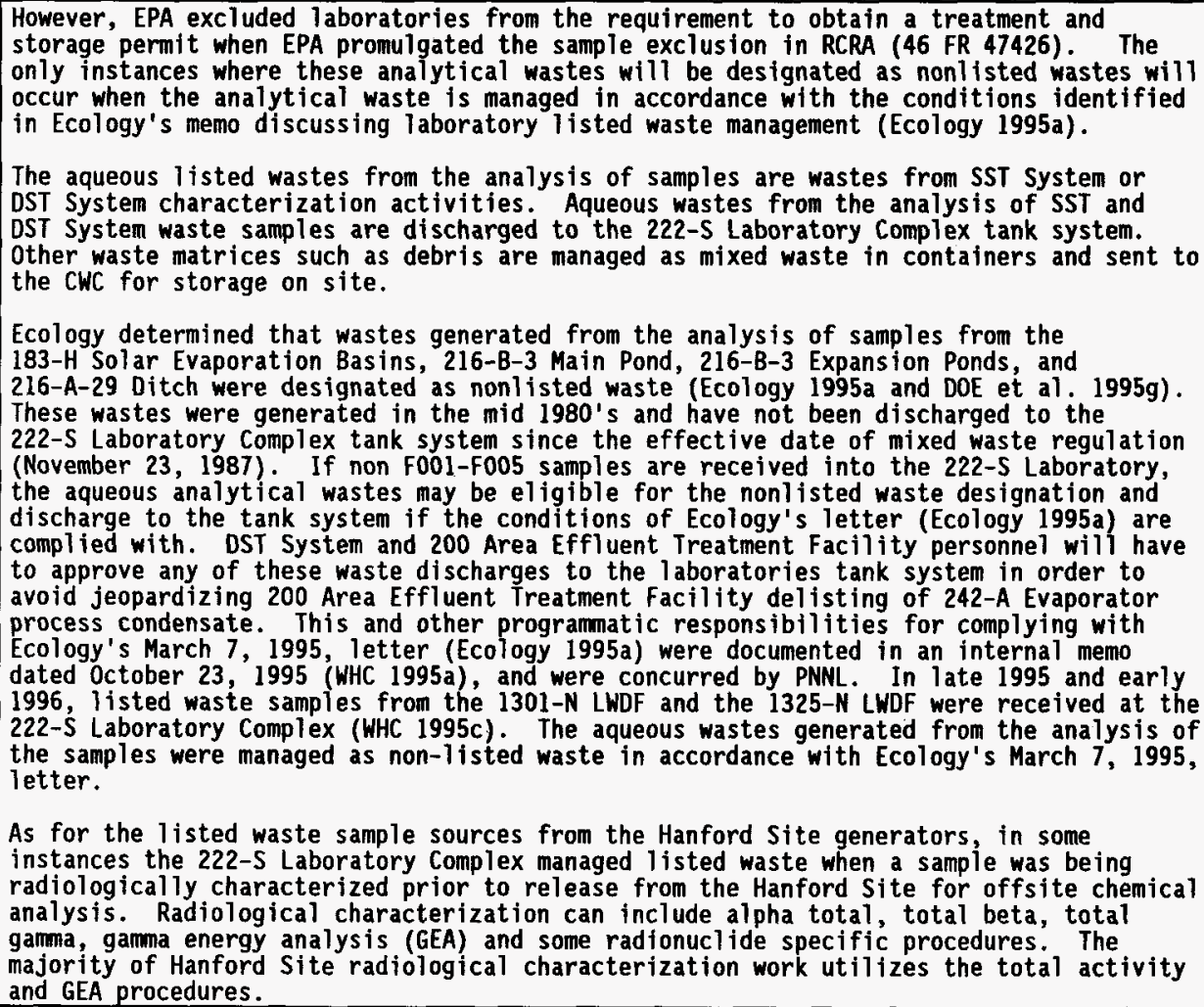 \\
\hline
\end{tabular}


Table 2. Listed Waste Management History at Treatment, Storage, and Disposal Units. (Sheet 13 of 31 )

\begin{tabular}{|c|c|}
\hline TSD Unit & Listed Waste History \\
\hline $\begin{array}{l}\text { 222-S LABORATORY } \\
\text { COMPLEX (cont.) }\end{array}$ & 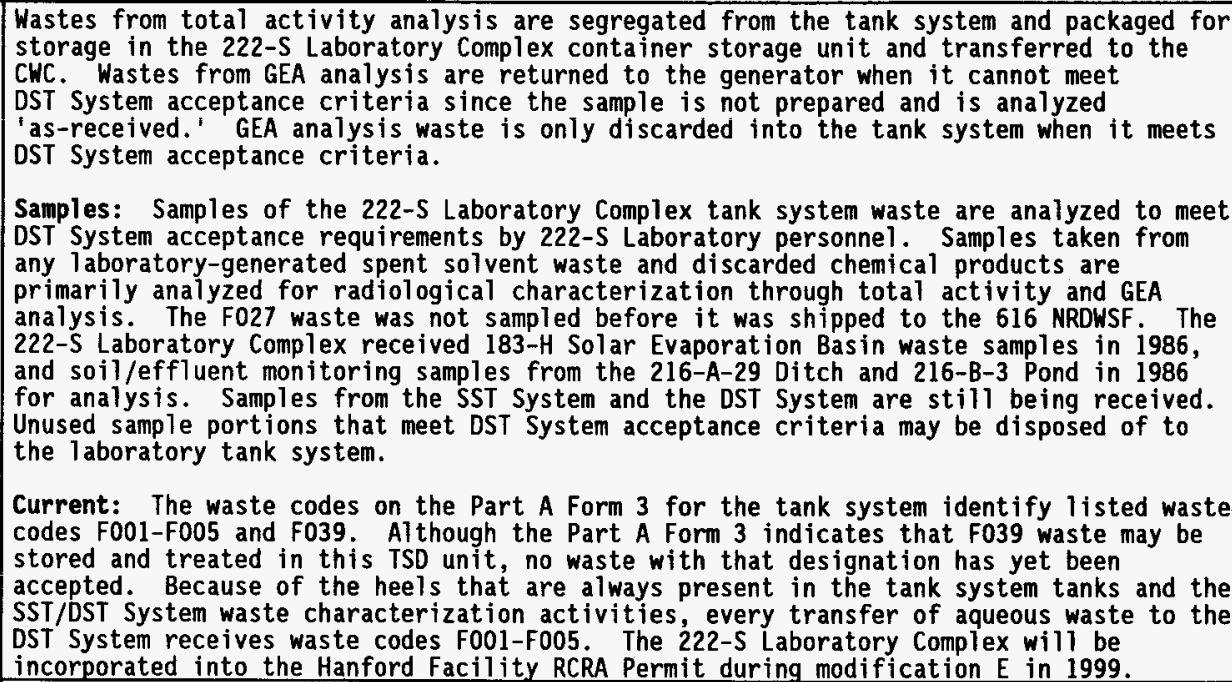 \\
\hline $\begin{array}{l}\text { 2727-S STORAGE } \\
\text { FACILITY }\end{array}$ & $\begin{array}{l}\text { Unit History: This TSD unit was the nonradioactive storage unit that pre-dates the } \\
616 \text { NRDWS. The unit managed listed waste in containers and staged them for offsite } \\
\text { disposal for three years from } 1983 \text { through } 1986 \text { (DOE 1992a). } \\
\text { Samples: Samples were not taken from the waste that was managed in the TSD unit. } \\
\text { Current: There are no } 1 \text { isted waste concerns or issues at this TSD unit. This unit was } \\
\text { clean closed on June } 27 \text {, } 1995 \text { and is included in the Hanford Facility RCRA Permit } \\
\text { Dangerous Waste Portion as Attachment } 17 \text {. }\end{array}$ \\
\hline
\end{tabular}


Table 2. Listed Waste Management History at Treatment, Storage, and Disposal Units. (Sheet 14 of 31 )

\begin{tabular}{|c|c|}
\hline TSD Unit & Listed Waste History \\
\hline $\begin{array}{l}\text { LIQUID EFFLUENT } \\
\text { RETENTION } \\
\text { FACILITY }\end{array}$ & 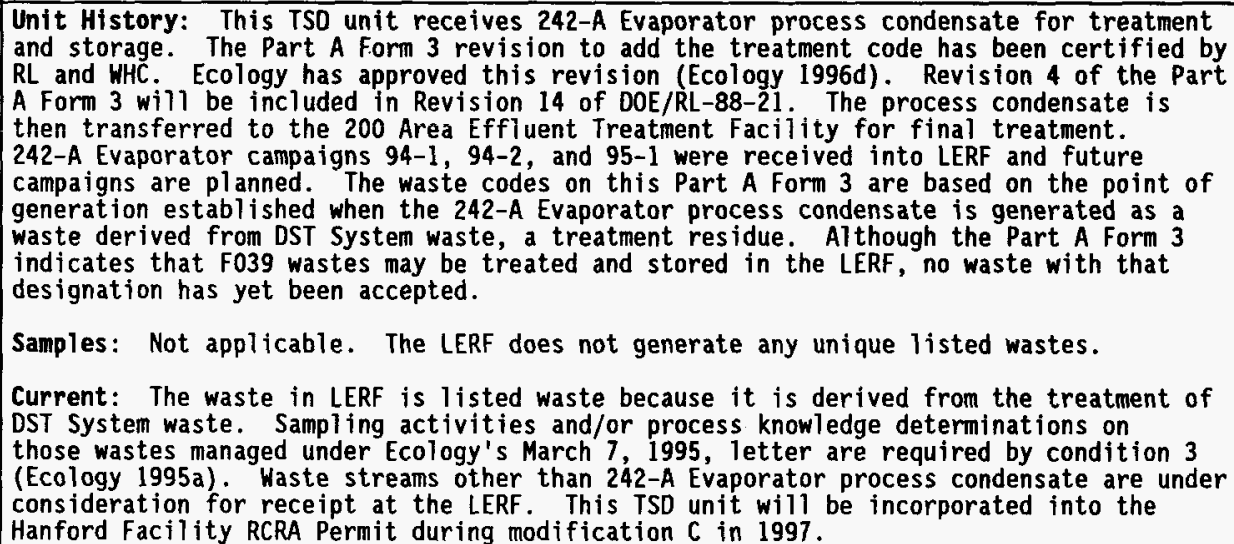 \\
\hline
\end{tabular}


Table 2. Listed Waste Management History at Treatment, Storage, and Disposal Units.

(Sheet 15 of 31 )

\begin{tabular}{|c|c|}
\hline TSD Unit & Listed Waste History \\
\hline $\begin{array}{l}\text { DOUBLE-SHELL TANK } \\
\text { SYSTEM }\end{array}$ & 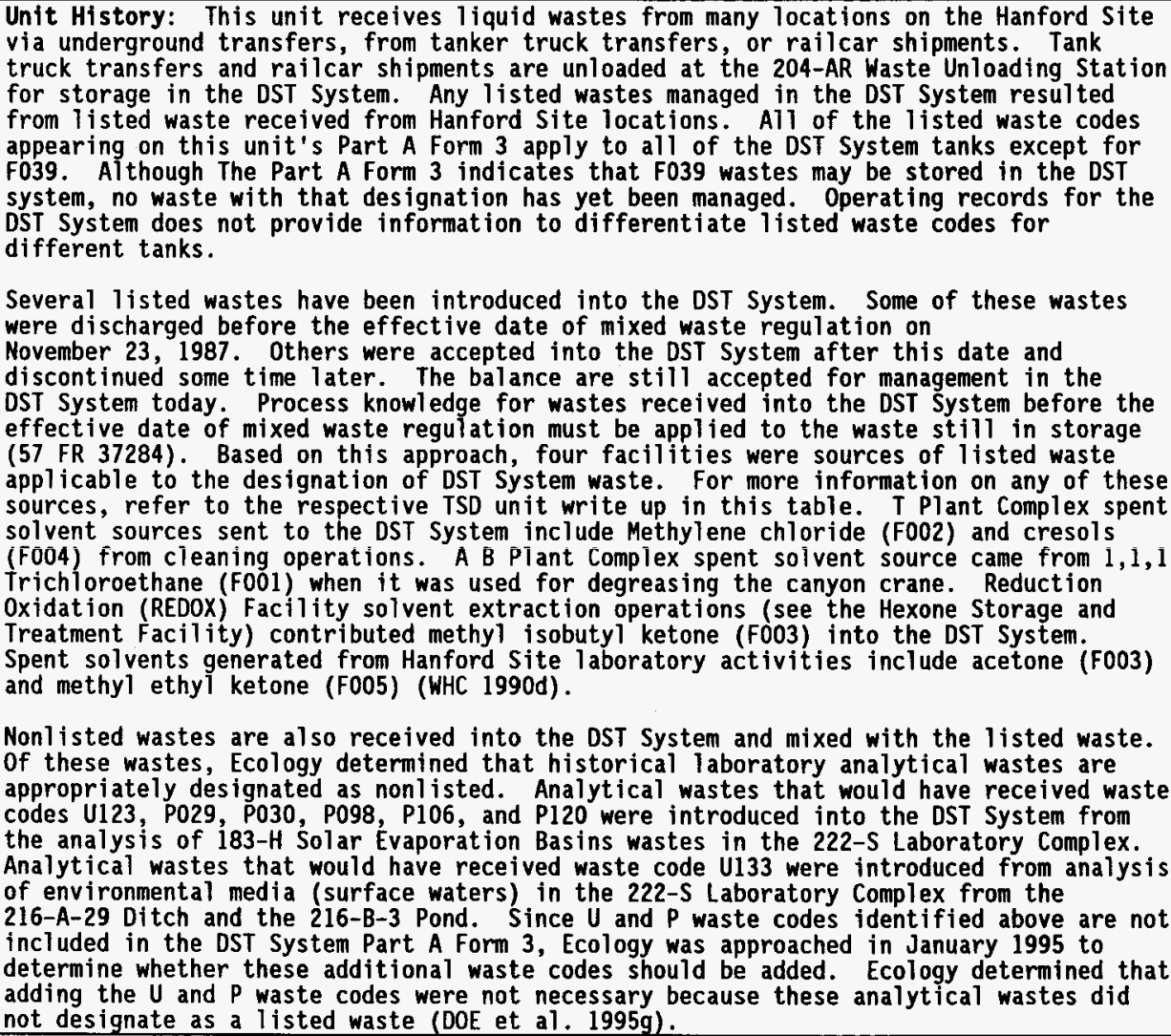 \\
\hline
\end{tabular}


Table 2. Listed Waste Management History at Treatment, Storage, and Disposal Units. (Sheet 16 of 31 )

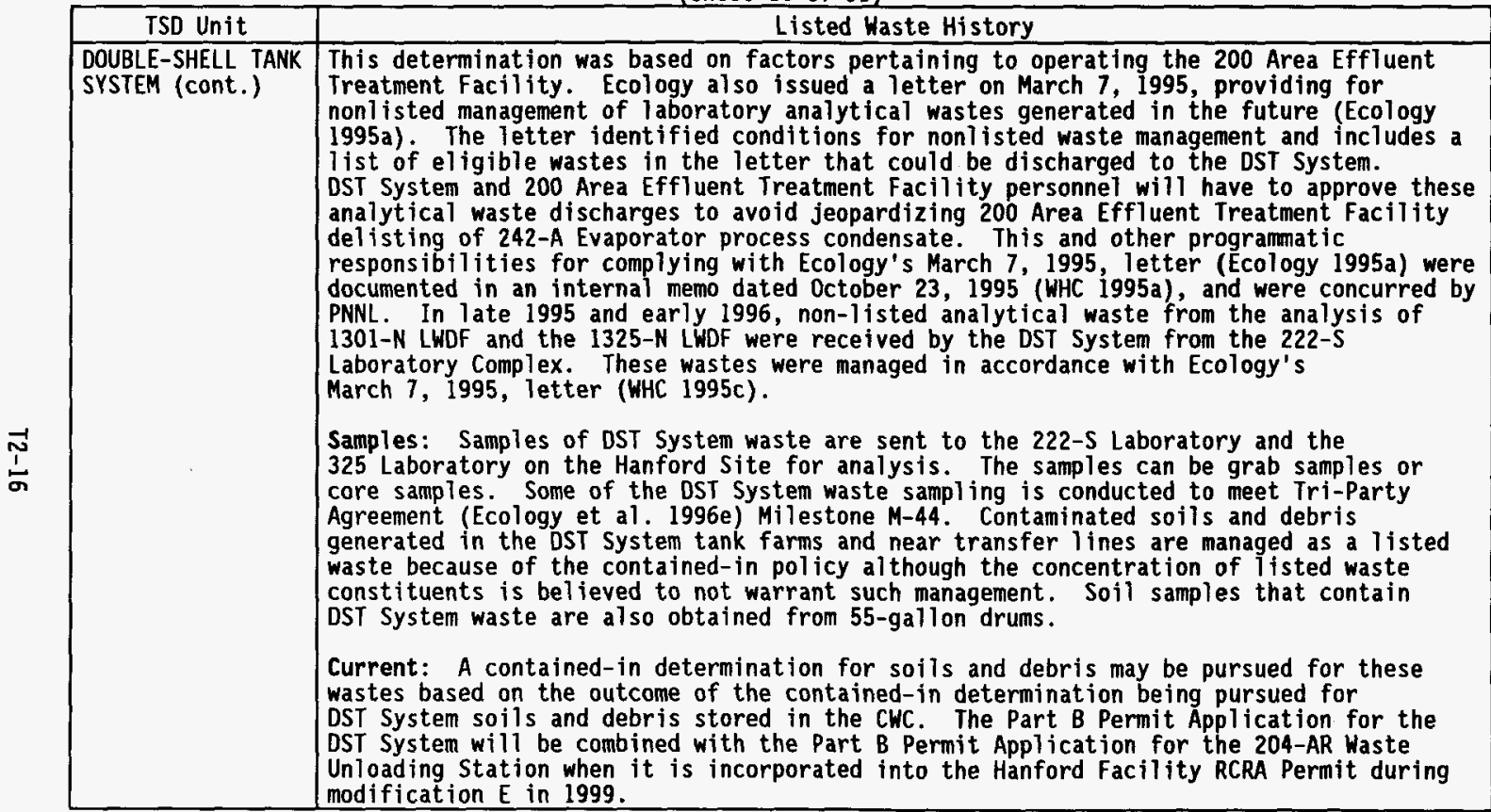




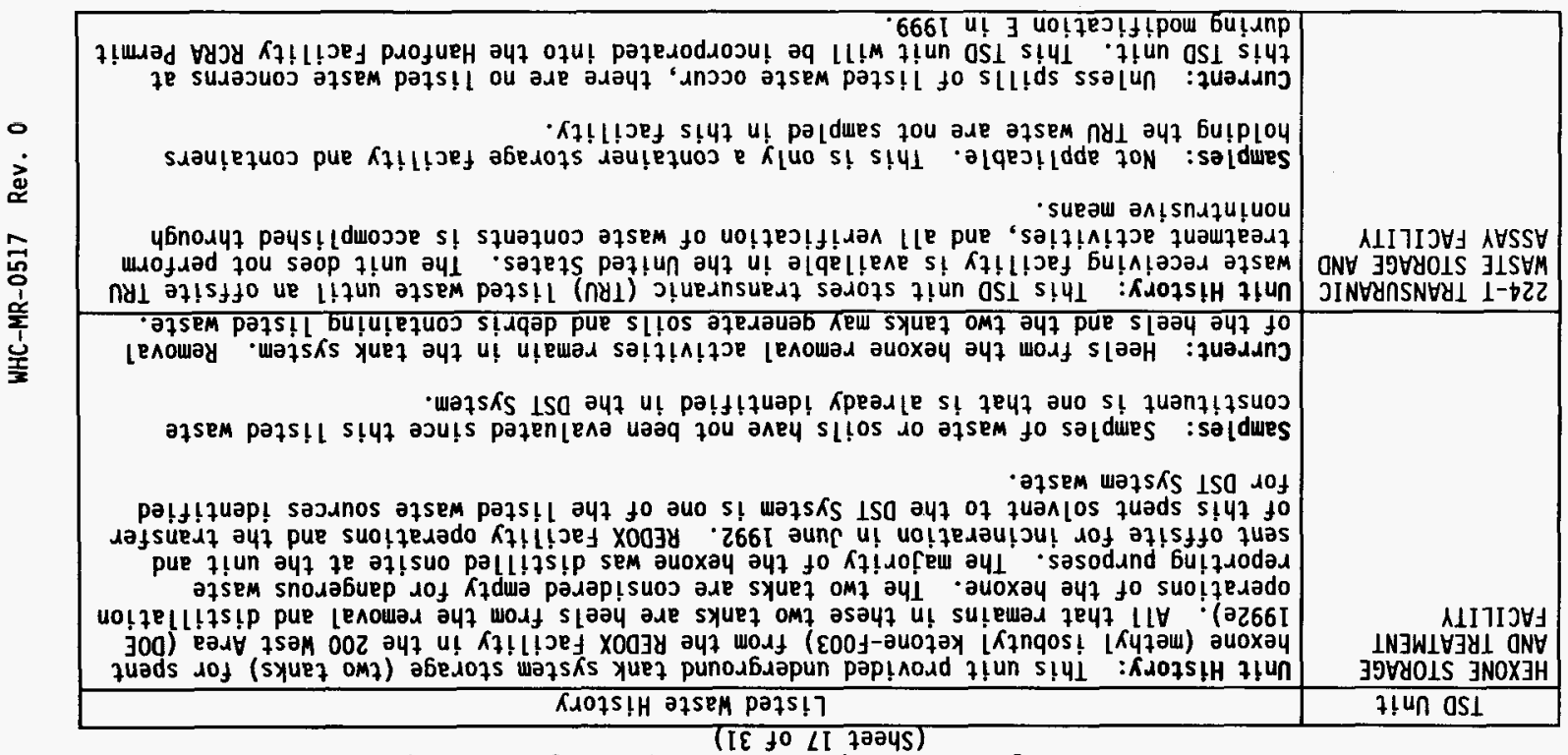

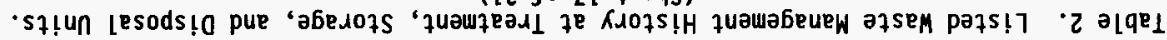


Table 2. Listed Waste Management History at Treatment, Storage, and Disposal Units.

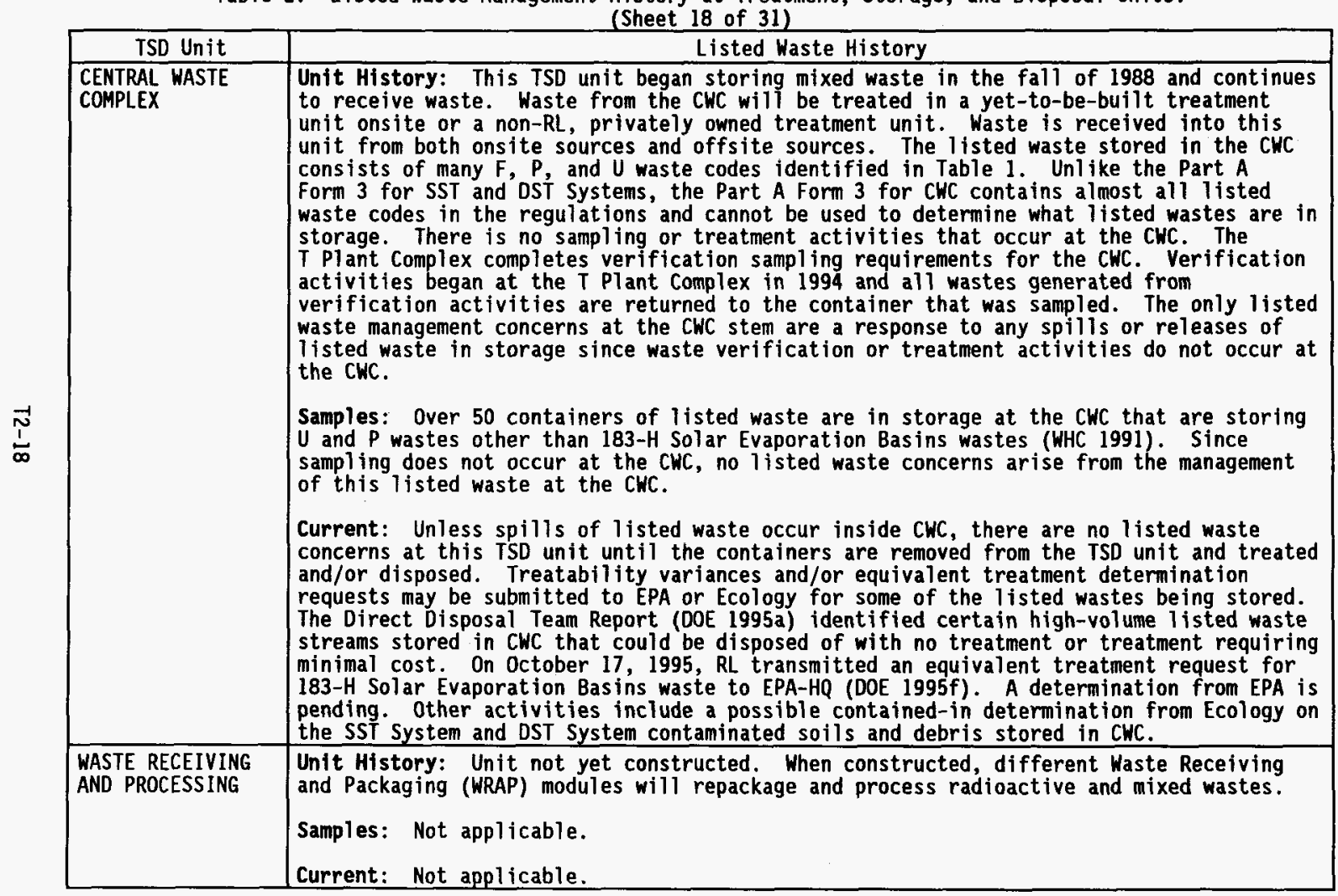


Table 2. Listed Waste Management History at Treatment, Storage, and Disposal Units.

\begin{tabular}{|c|c|}
\hline TSD Unit & Listed Waste History \\
\hline $\begin{array}{l}\text { SINGLE-SHELL TANK } \\
\text { SYSTEM }\end{array}$ & 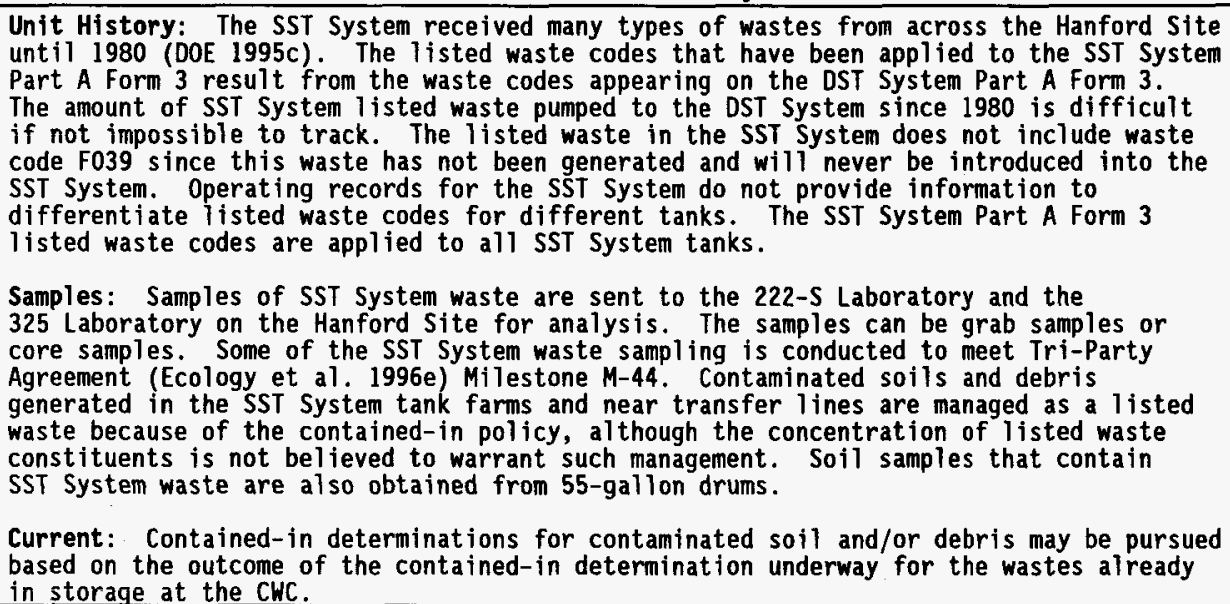 \\
\hline $\begin{array}{l}\text { 207-A SOUTH } \\
\text { RETENTION BASIN }\end{array}$ & $\begin{array}{l}\text { Unit History: This TSD unit received } 242-A \text { Evaporator process condensate prior to } \\
\text { construction of the LERF. The process COndensate was discharged from the 207-A South } \\
\text { Retention Basins to the 216-A-37-1 Crib (DOE 1988). } \\
\text { Samples: Samples of waste or soil from this unit have not been evaluated since the } \\
\text { waste is derived from OST System waste. } \\
\text { Current: The concrete basin and any liquids that collect in the basins from } \\
\text { precipitation should not be expected to contain any listed waste. Concurrence in this } \\
\text { belief has not been documented through Ecology. }\end{array}$ \\
\hline
\end{tabular}


Table 2. Listed Waste Management History at Treatment, Storage, and Disposal Units. (Sheet 20 of 31)

\begin{tabular}{|c|c|}
\hline TSD Unit & Listed Waste History \\
\hline $\begin{array}{l}\text { LOW-LEVEL BURIAL } \\
\text { GROUNDS }\end{array}$ & $\begin{array}{l}\text { Unit History: This disposal unit has disposed of waste that could be designated as } \\
\text { listed waste if it is actively managed after the effective date of regulation (57 FR } \\
37284 \text { ). The amount of waste disposed has not been evaluated for this document. Burial } \\
\text { records indicate a wide variety of listed waste that may require evaluation if non-TRU } \\
\text { wastes are ever excavated and actively managed. The new disposal trenches (currently } \\
\text { two) meeting minimum technology requirements for RCRA Subtitle C disposal will receive } \\
\text { mixed waste for disposal in compliance with all applicable requirements. F039 leachate } \\
\text { will be generated by the new mixed waste trenches at some time in the future. } \\
\text { Waste verification activities for wastes received into the LLBG are performed at the } \\
\text { T Plant Complex. There are no sampling or treatment activities occurring at the LLBG. } \\
\text { The only listed waste management concerns at the LLBG stem from responding to spills or } \\
\text { releases of listed waste. } \\
\text { Samples: Samples taken of waste, soil, or groundwater from this unit has not been } \\
\text { evaluated since iisted waste has not been actively managed. } \\
\text { Current: Suspect-TRU and TRU retrieval activities have commenced. These excavated } \\
\text { wastes will be managed as nondangerous wastes until information is generated from } \\
\text { nondestructive examination at the } 224-T \text { Transuranic Waste Storage and Assay Facility and } \\
\text { from the evaluation of available records. Other wastes in the Low-Level Burial Grounds } \\
\text { are not planned for retrieval. }\end{array}$ \\
\hline $216-A-37-1$ CRIB & $\begin{array}{l}\text { Unit History: This disposal unit began operation in March } 1977 \text { and received } \\
242-A \text { Evaporator process condensate from the 207-A South Retention Basins until } \\
\text { Apri] } 1989 \text { (DOE 1988). } \\
\text { Samples: Samples of soils or process waters have not been evaluated since these wastes } \\
\text { are derived from DST System wastes. } \\
\text { Current: Listed waste contained-in evaluations on the soils have not been performed. }\end{array}$ \\
\hline
\end{tabular}


Table 2. Listed Waste Management History at Treatment, Storage, and Disposal Units.

\begin{tabular}{|c|c|}
\hline TSD Unit & Listed Waste History \\
\hline $\begin{array}{l}\text { 216-B-3 EXPANSION } \\
\text { POND }\end{array}$ & $\begin{array}{l}\text { Unit History: This TSD unit received effluents from PUREX, B Plant, and other } 200 \text { East } \\
\text { Area Hanford Site facilities and consists of three lobes of the } 216-\mathrm{B}-3 \text { Pond ( } 3 \mathrm{~A}, 3 \mathrm{~B} \text {, } \\
\text { and } 3 \mathrm{C} \text { ) (DOE 1994C). The } 216-\mathrm{A}-29 \text { Ditch discharged to the } 216-\mathrm{B}-3 \text { Pond unt il it was } \\
\text { interim stabilized. The only } 1 \text { isted waste received into this unit was a one-time-only } \\
\text { discharge that occurred in July } 1986 \text { from the } 216-\mathrm{A}-29 \text { Ditch. This TSD unit was clean } \\
\text { closed on June } 27 \text {, 1995, and is included in the Hanford Facility RCRA Permit Dangerous } \\
\text { Waste portion as Attachment } 23 \text {. }\end{array}$ \\
\hline & $\begin{array}{l}\text { Samples: Soil and effluent samples have been taken from this unit for environmental } \\
\text { monitoring and closure activities. Annual reports for Environmental Surveillance } \\
\text { activities from } 1986 \text { to } 1987 \text { and beyond showed that water, vegetation, and sediment } \\
\text { samples were taken and analyzed by the } 222-5 \text { Laboratory Complex. RCRA closure sampling } \\
\text { activities in } 1989 \text { showed that hydrazine could not be detected. }\end{array}$ \\
\hline & $\begin{array}{l}\text { Current: There are no listed waste issues associated with this TSD unit. Clean closure } \\
\text { of this TSD unit was based on the chemical nature of hydrazine and the } 1989 \text { sampling } \\
\text { activities. }\end{array}$ \\
\hline 216-A-29 DITCH & $\begin{array}{l}\text { Unit History: This unit received chemical sewer effluents from the PUREX Facility. } \\
\text { The listed waste discharged to this unit consists of a single spil1 to the unit of a } \\
\text { unused commercial chemical product. The spill occurred on July } 7,1986 \text {, and consisted } \\
\text { of a sole active ingredient, a hydrazine-water solution (RH0 1986C). The effluent from } \\
\text { this unit is sent to the } 216-\mathrm{B}-3 \text { Pond via the } 216-\mathrm{B}-3-3 \text { Ditch. }\end{array}$ \\
\hline & $\begin{array}{l}\text { Samples: Soil and effluent samples have been taken from this unit for environmental } \\
\text { monitoring purposes. Annual reports for Environmental Surveillance act ivities from } 1986 \\
\text { to } 1987 \text { and beyond showed that water, vegetation, and sediment samples were taken and } \\
\text { analyzed by the } 222-5 \text { Laboratory Complex. }\end{array}$ \\
\hline & $\begin{array}{l}\text { Current: This TSD unit will be incorporated into the Hanford Facility RCRA Permit } \\
\text { during modification } F \text { in } 2000 \text {. Clean closure of the TSD unit is expected based on the } \\
\text { chemical nature of hydrazine and the } 1989 \text { sampling activities at the } 216-B-3 \text { Pond. } \\
\text { There is no reason to believe that soil or surface water samples contain } 1 \text { isted waste. } \\
\text { A request for a contained-in determination will be addressed in closure documentation. }\end{array}$ \\
\hline
\end{tabular}


Table 2. Listed Waste Management History at Treatment, Storage, and Disposal Units. (Sheet 22 of 31)

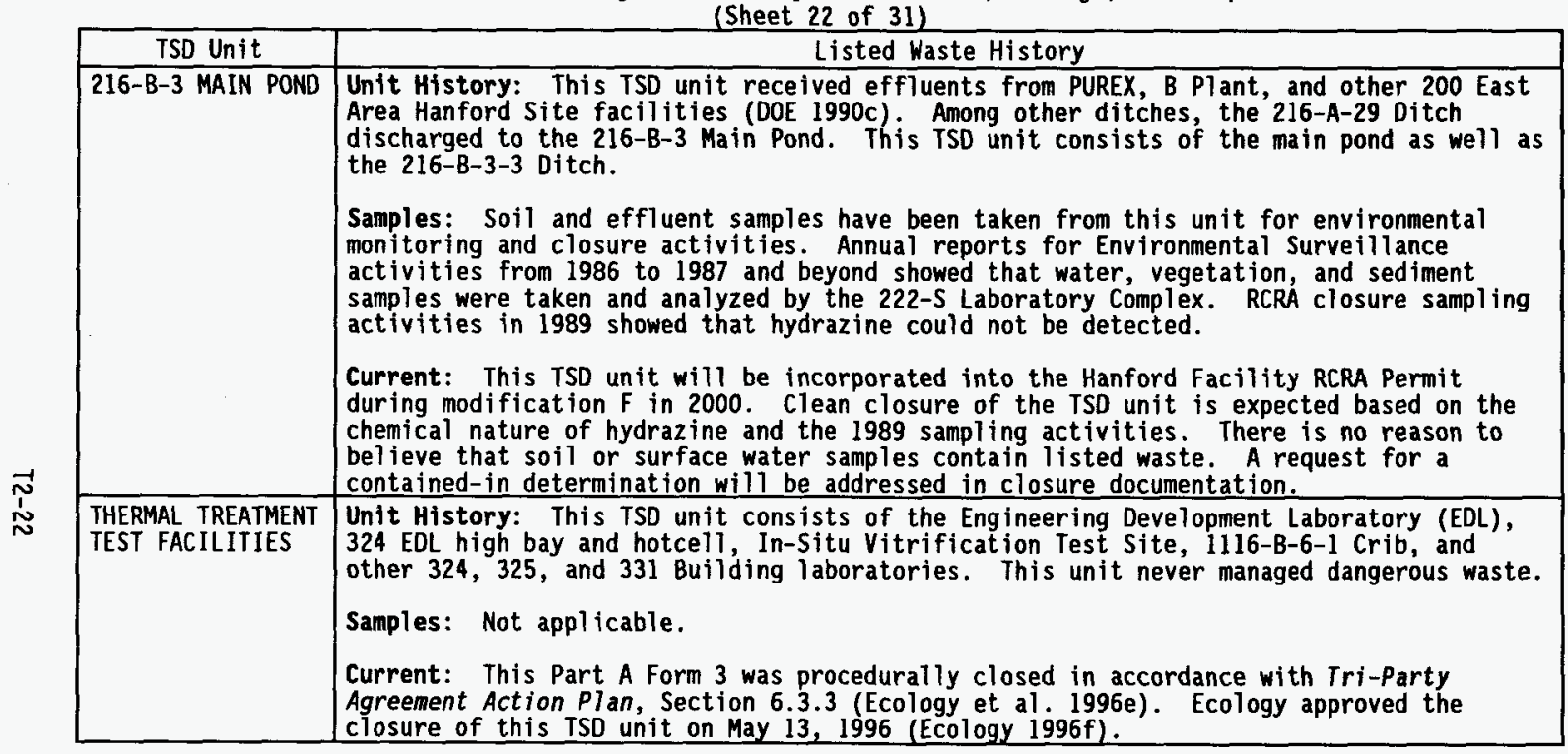


Table 2. Listed Waste Management History at Treatment, Storage, and Disposal Units.

\begin{tabular}{|c|c|}
\hline TSD Unit & \begin{tabular}{|l} 
Listed Waste History \\
\end{tabular} \\
\hline & $\begin{array}{l}\text { Concrete and soil samples also were obtained during a separate sampling event; however, } \\
\text { these samples do not have any listed waste concerns associated with them. This is } \\
\text { because of closure plan language (page 3-5) that states: "Based on the spil11 } \\
\text { scenario.... it is likely that little, if any, waste was discharged from the } 300 \text { Area } \\
\text { Solvent Evaporator (ASE) when it was inadvertently filled with water. . . from the leaky } \\
\text { steam heating coil system [and overflowed]. A few sentences later: "Such small } \\
\text { quantities of solvent evaporator volatile components (if any) would be likely to have } \\
\text { since evaporated from the soil..." Therefore, the soil and concrete samples did not } \\
\text { contain any listed waste when analyzed. }\end{array}$ \\
\hline & $\begin{array}{l}\text { Current: There are no } 1 \text { isted waste issues associated with this unit. The TSO unit was } \\
\text { clean closed on June } 27,1995 \text {. }\end{array}$ \\
\hline $\begin{array}{l}300 \text { AREA WASTE } \\
\text { ACID TREATMENT } \\
\text { SYSTEM }\end{array}$ & $\begin{array}{l}\text { Unit History: This Part A Form } 3 \text { has two units grouped together: (1) The } 300 \text { Area WATS } \\
\text { and (2) the } 311 \text { Tanks. Listed waste codes P120 and U123 are identified on } \\
\text { Revision } 3 \text { of the Part A Form } 3 \text { and are only associated with the } 300 \text { Area WATS unit. } \\
\text { Tanks } 40 \text { and } 50 \text { are the two tanks that make up the } 311 \text { Tanks entry on the Part A Form } 3 \\
\text { that received wastes from the } 300 \text { Area WATS. Although } 1 \text { isted waste codes P120 and U123 } \\
\text { appear in this Part A Form } 3 \text {, these listed wastes were never managed in either of these } \\
\text { units upon the effective day of the RCRA regulations for nonradioactive wastes in } 1980 \text {. }\end{array}$ \\
\hline
\end{tabular}




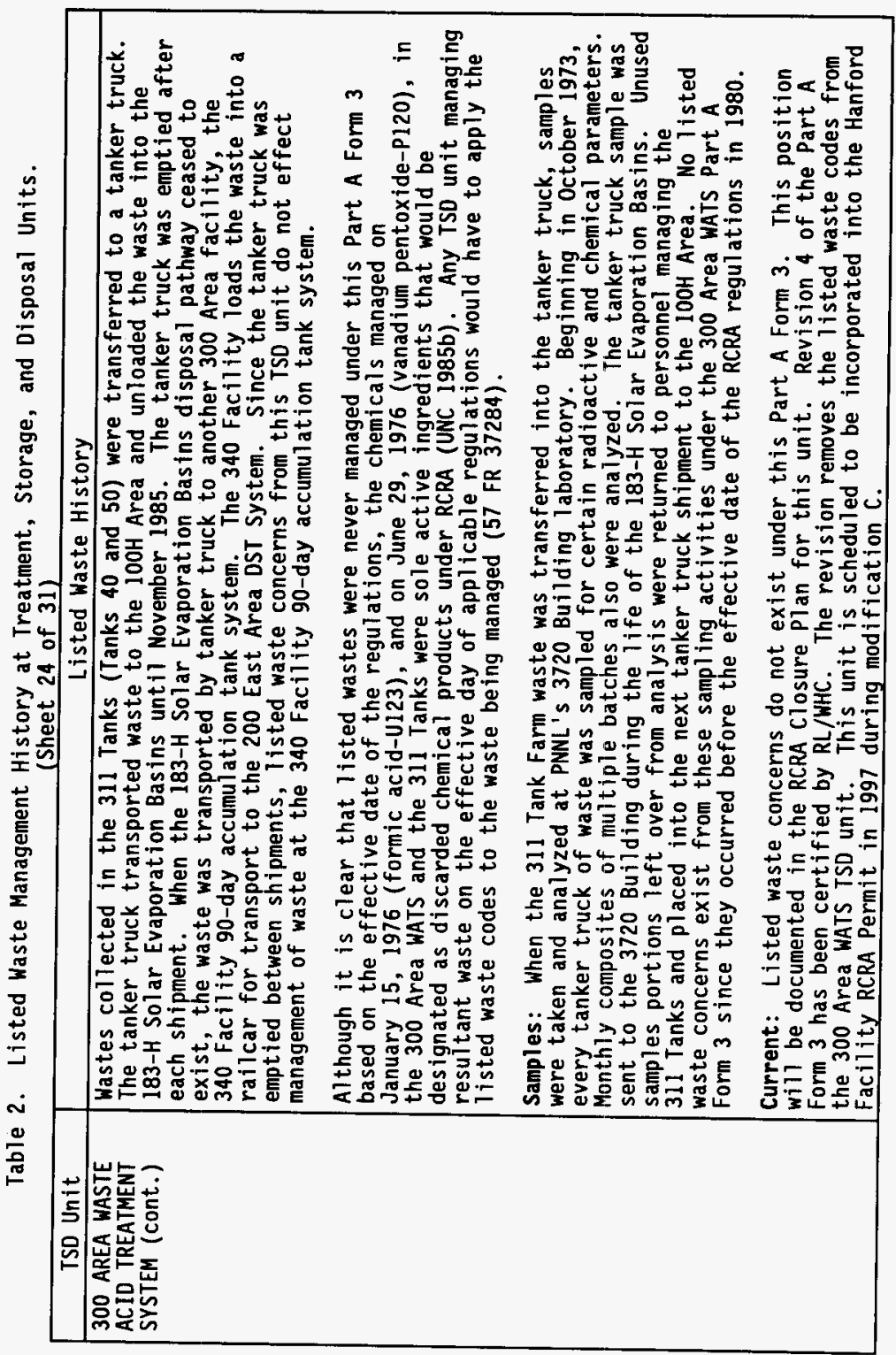


Table 2. Listed Waste Management History at Treatment, Storage, and Disposal Units. (Sheet 25 of 31 )

\begin{tabular}{|c|c|}
\hline TSD Unit & Listed Waste History \\
\hline $\begin{array}{l}325 \text { HAZARDOUS } \\
\text { WASTE TREATMENT } \\
\text { UNITS }\end{array}$ & 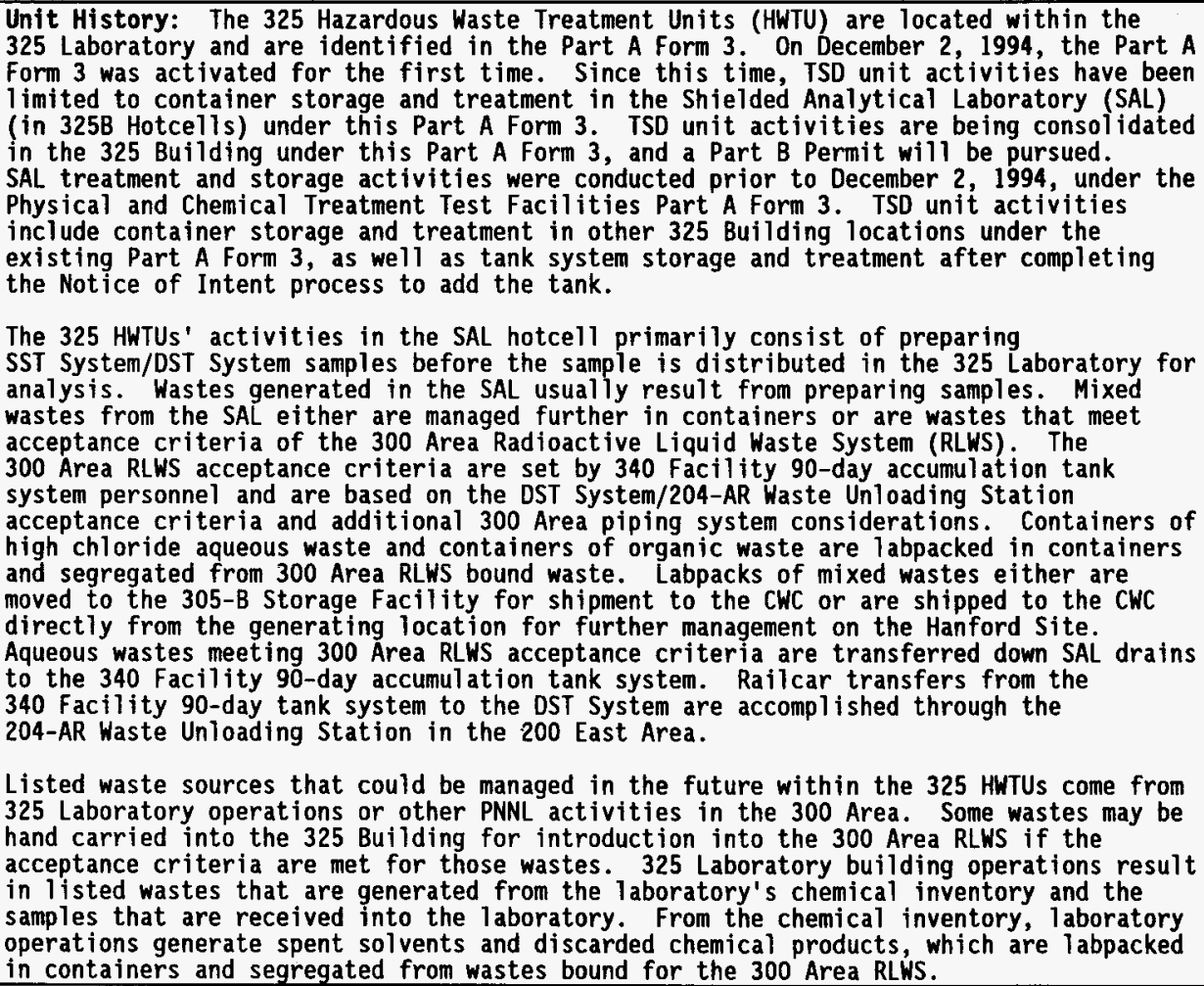 \\
\hline
\end{tabular}


Table 2. Listed Waste Management History at Treatment, Storage, and Disposal Units. (Sheet 26 of 31 )

\begin{tabular}{|c|c|}
\hline TSD Unit & Listed Waste History \\
\hline $\begin{array}{l}325 \text { HAZARDOUS } \\
\text { WASTE TREATMENT } \\
\text { UNITS (cont.) }\end{array}$ & $\begin{array}{l}\text { The only listed wastes discharged from portions of the HWTU to the } 300 \text { Area RLWS under } \\
\text { this Part A Form } 3 \text { are those FOO1-FOO5 wastes that meet the acceptance criteria. These } \\
\text { aqueous wastes are analytical wastes generated from SST System or DST System } \\
\text { characterization activities. } \\
\text { Samples: Any samples taken of SAL-generated wastes are analyzed in the } 325 \text { Laboratory. } \\
\text { SAL wastes are sampled to confirm that acceptance criteria are met for either the } \\
300 \text { Area RLWS or CWC. } \\
\text { Current: Listed waste is managed in containers in the SAL. Only when SAL waste is } \\
\text { discharged to the } 300 \text { Area RLWS via a building drain will the } 1 \text { isted waste enter the } \\
\text { tank being added to this Part A Form } 3 \text {. When other treatment and storage activities are } \\
\text { activated under this Part A Form } 3 \text {, listed waste management will increase and may } \\
\text { include management of the spent soivent and discarded chemical products generated within } \\
\text { the } 325 \text { Laboratory or other } 1 \text { isted waste samples received by personne } 1 \text { at the } \\
325 \text { Laboratory. }\end{array}$ \\
\hline $\begin{array}{l}\text { BIOLOGICAL } \\
\text { TREATMENT TEST } \\
\text { FACILITIES }\end{array}$ & $\begin{array}{l}\text { Unit History: This TSD unit Part A Form } 3 \text { lists the } 324,325 \text {, and } 331 \text { Buildings as } \\
\text { permitted locations. This unit never managed any dangerous waste. } \\
\text { Samples: Not applicable. } \\
\text { Current: This Part A Form } 3 \text { wi11 be procedurally closed in accordance with Tri-Party } \\
\text { Agreement Action Plan, Section } 6.3 .3 \text { (Ecology et al. 1996e). }\end{array}$ \\
\hline $\begin{array}{l}\text { PHYSICAL \& } \\
\text { CHEMICAL } \\
\text { TREATMENT TEST } \\
\text { FACILITIES }\end{array}$ & $\begin{array}{l}\text { Unit History: This TSD unit Part A Form } 3 \text { lists three locations where treatment and } \\
\text { storage in containers could occur: two in the } 324 \text { Building and one in the } 325 \text { Building. } \\
\text { TSD unit activities have only occurred in one location under this Part A Form } 3 \text { in the } \\
325 \text { Building SAL (in } 325 B \text { Hotcells). PNN Used this Part A Form } 3 \text { to treat SAL hotcell } \\
\text { wastes until December } 2 \text {, } 1994 \text {, when the } 325 \text { HWTU Part A Form } 3 \text { was activated. See the } \\
325 \text { HWTU discussion in this table for a description of the SAL activities. SST System } \\
\text { and DST System samples have been the only } 1 \text { isted wastes managed under this Part A } \\
\text { Form } 3 \text {. All other listed waste managed within the } 325 \text { Building have been managed under } \\
\text { the generator provisions of WAC } 173-303 \text { and not under any PNNL Part A Form } 3 \text {. } \\
\text { Samples: Any samples taken of SAL-generated wastes were analyzed in the } 325 \text { Laboratory. } \\
\text { SAL wastes were sampled to meet either } 300 \text { Area RLWS or CWC acceptance criteria. } \\
\text { Current: The TSD unit has been inactive since December } 2 \text {, 1994. Since waste management } \\
\text { activities under the Part A Form } 3 \text { were transferred to the } 325 \text { HWTU Part A Form } 3 \text {, } \\
\text { Ecology approved the closure of this TSD unit on May } 13 \text {, 1996 (Ecology 1996f). }\end{array}$ \\
\hline
\end{tabular}


Tabie 2. Listed Waste Management History at Treatment, Storage, and Disposal Units.

\begin{tabular}{|c|c|}
\hline TSD Unit & Listed Waste History \\
\hline $\begin{array}{l}\text { 303-K STORAGE } \\
\text { UNIT }\end{array}$ & $\begin{array}{l}\text { Unit HIstory: This TSD unit is a container storage unit. This TSD unit managed spent } \\
\text { solvents from the fuel fabrication activities in the } 300 \text { Area. } 57 \text { drums of absorbed } \\
\text { spent solvents were managed on this pad until } 1993 \text {. These drums are now stored in the } \\
\text { CWC as mixed waste. } \\
\text { Samples: The drums of waste were sampled in order to meet CWC acceptance criteria. } \\
\text { The samples were sent offsite for analysis. } \\
\text { Current: There is no listed waste managed in the TSD unit now, and the unit is expected } \\
\text { to be clean closed. The TSD unit will be incorporated into the Hanford Facility RCRA } \\
\text { Permit in } 1997 \text { during modification C. }\end{array}$ \\
\hline $\begin{array}{l}\text { 305-B STORAGE } \\
\text { FACILITY }\end{array}$ & $\begin{array}{l}\text { Unit History: This TSD unit has obtained its Part B Permit at the same time as the } \\
616 \text { NRDWSF. It is included in the Hanford Facility RCRA Permit Dangerous Waste portion } \\
\text { as Attachment } 18 \text {. Wastes generated from PNNL operations are managed through the } \\
305-B \text { Storage Unit. The } 305-B \text { Storage unit is a container storage unit. Listed wastes } \\
\text { have been routinely managed through this building and the Part A Form } 3 \text { has almost all } \\
\text { possible listed waste codes on it. } \\
\text { Samples: Not applicable with the exception of verification activities required by the } \\
\text { Part B Permit. Verification activities result in samples being analyzed at } 305-B \text { and } \\
\text { a11 wastes are placed back into the parent container. } \\
\text { Current: Unless spills of listed waste occur that are not properly cleaned up, there } \\
\text { are no listed waste concerns at this TSD unit. }\end{array}$ \\
\hline $\begin{array}{l}332 \text { STORAGE } \\
\text { FACILITY }\end{array}$ & $\begin{array}{l}\text { Unit History: This TSD unit has never managed dangerous waste and the Part A Form } 3 \\
\text { will undergo procedural closure. } \\
\text { Samples: Not applicable. } \\
\text { Current: Not applicable. }\end{array}$ \\
\hline
\end{tabular}


Table 2. Listed Waste Management History at Treatment, Storage, and Disposal Units. (Sheet 28 of 31 )

\begin{tabular}{|c|c|}
\hline TSD Unit & Listed Waste History \\
\hline $\begin{array}{l}300 \text { AREA PROCESS } \\
\text { TRENCHES }\end{array}$ & 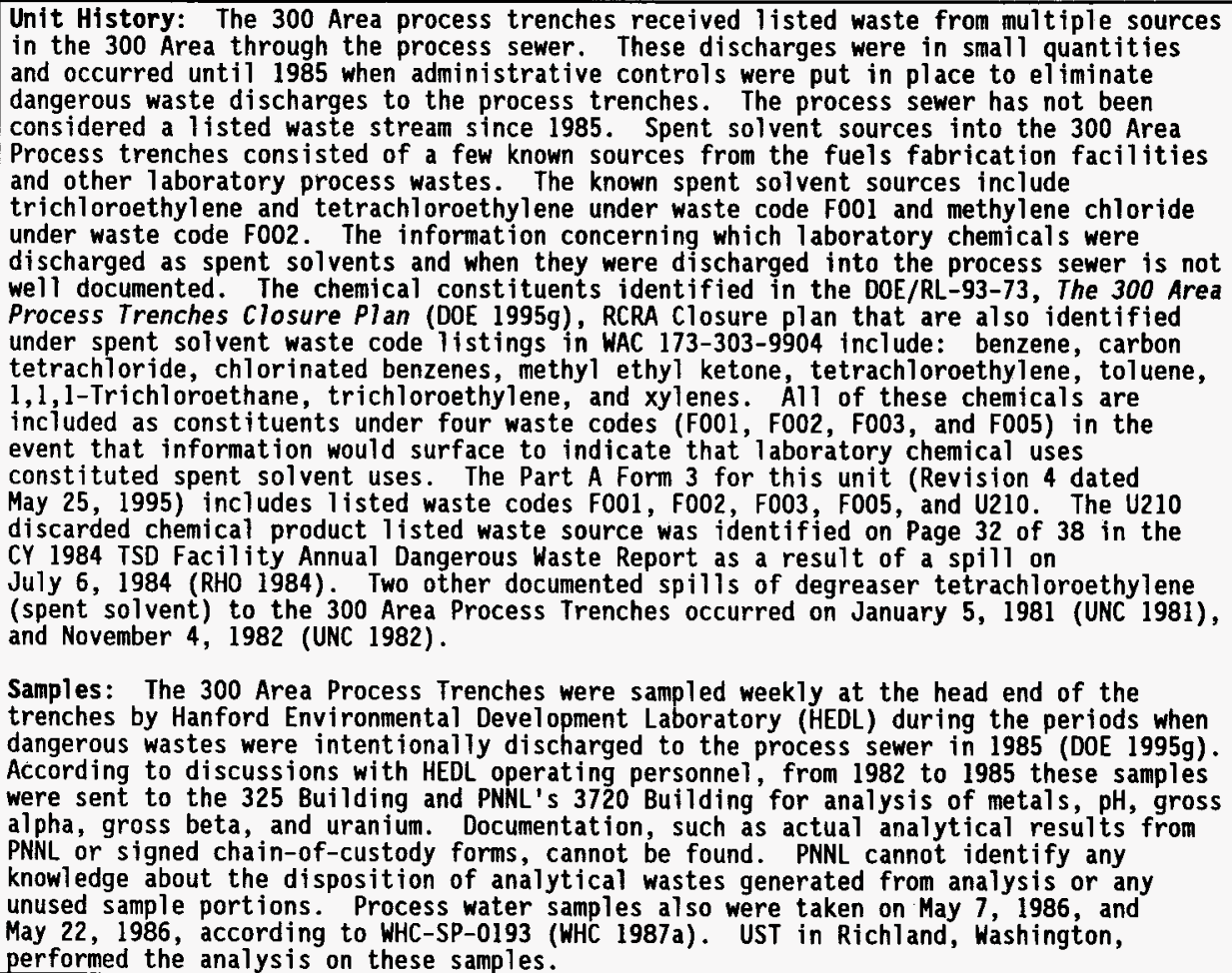 \\
\hline
\end{tabular}


Table 2. Listed Waste Management History at Treatment, Storage, and Disposal Units. (Sheet 29 of 31 )

\begin{tabular}{|c|c|}
\hline TSD Unit & Listed Waste History \\
\hline $\begin{array}{l}300 \text { AREA PROCESS } \\
\text { TRENCHES (cont.) }\end{array}$ & 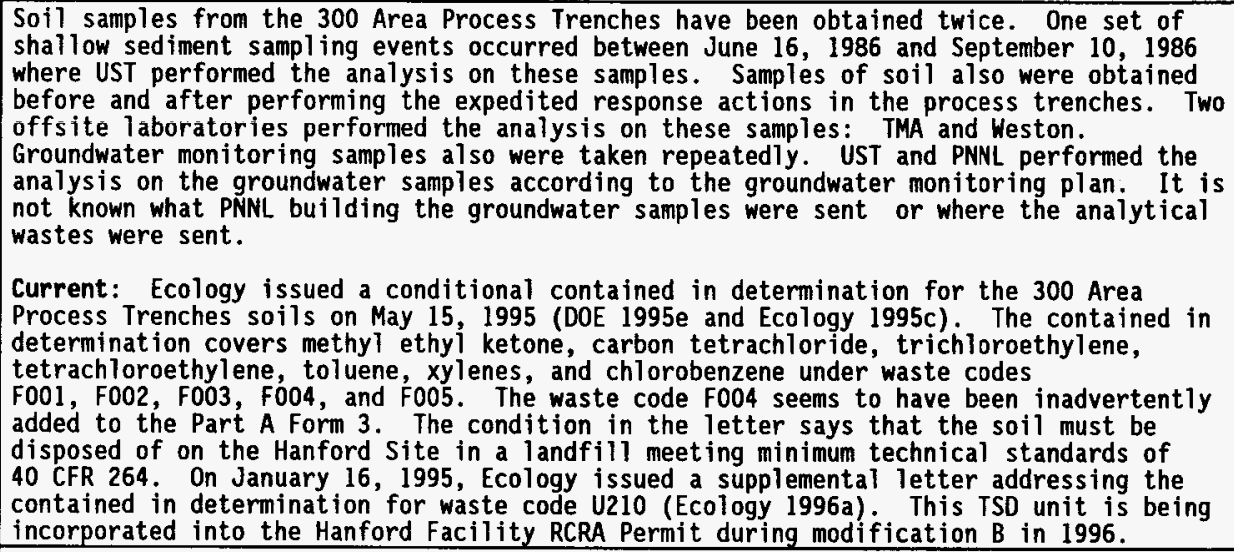 \\
\hline $\begin{array}{l}\text { HANFORD PATROL } \\
\text { ACADEMY } \\
\text { DEMOLITION SITES }\end{array}$ & $\begin{array}{l}\text { Unit History: This TSD unit detonated discarded chemical products during its lifetime. } \\
\text { The listed waste that was detonated is reflected on the Part A Form } 3 \text { for the unit. On } \\
\text { March } 12 \text {, } 1987 \text {, a demolition failure occurred such that contaminated soil was placed } \\
\text { into } 30-\text {-gallon drums and sent directly offsite (not through } 616 \text { NRDWSF) for disposal. } \\
\text { This TSD unit has been added as a Part } V \text { unit to the Hanford Facility RCRA Permit. } \\
\text { DOE/RL-92-39, Hanford Patrol Academy Demolition Sites Closure Plan, Revision } 1 \text {, } \\
\text { (DOE 1994b) is Attachment } 24 \text { to the Hanford Facility RCRA Permit. } \\
\text { Samples: On September } 26,1994 \text {, soil samples were acquired from the TSD unit locations } \\
\text { and sent offsite to Lockheed for analysis to support closure activities. Samples also } \\
\text { were obtained from soil at the TSD unit as provided in Appendix } 3 A \text { to the Hanford Patrol } \\
\text { Academy Demolittion Sites Closure Plan (DOE 1994b). The laboratories used for these } \\
\text { samples include the Hanford Environmental Health Foundation (HEHF) laboratory in } \\
\text { Richland, Washington, as well as Northwest Enviroservice in Seattle, Washington. } \\
\text { Current: There are no } 1 \text { isted waste concerns at this TSD unit. The TSD unit has been } \\
\text { clean closed as of October } 26 \text {, 1995. Clean closure was based on information pertaining } \\
\text { to the efficiency by which chemicals are destroyed through detonation and the time that } \\
\text { has elapsed from the last event at the TSD unit. }\end{array}$ \\
\hline
\end{tabular}


Table 2. Listed Waste Management History at Treatment, Storage, and Disposal Units.

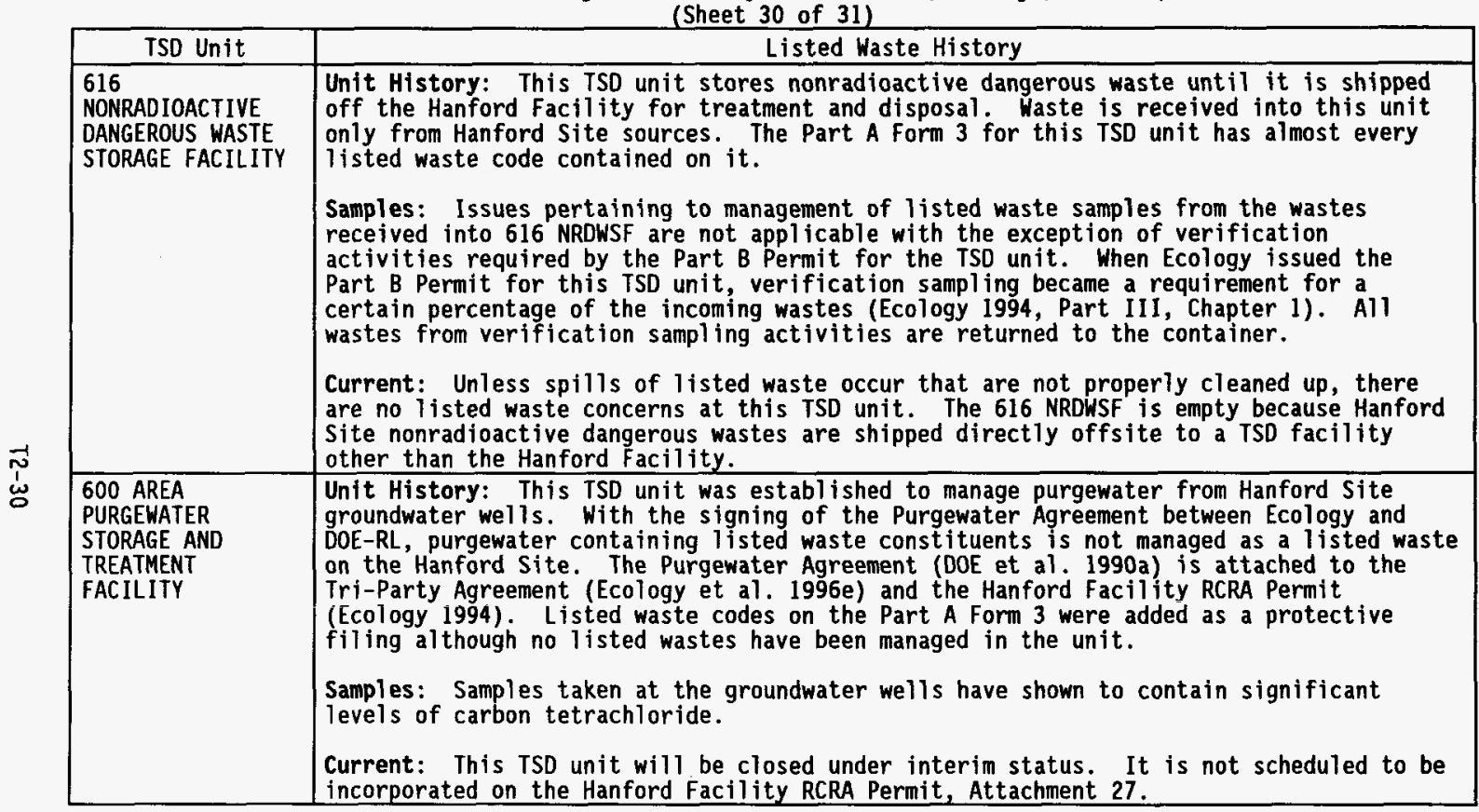


Table 2, Listed Waste Management History at Treatment, Storage, and Disposal Units.

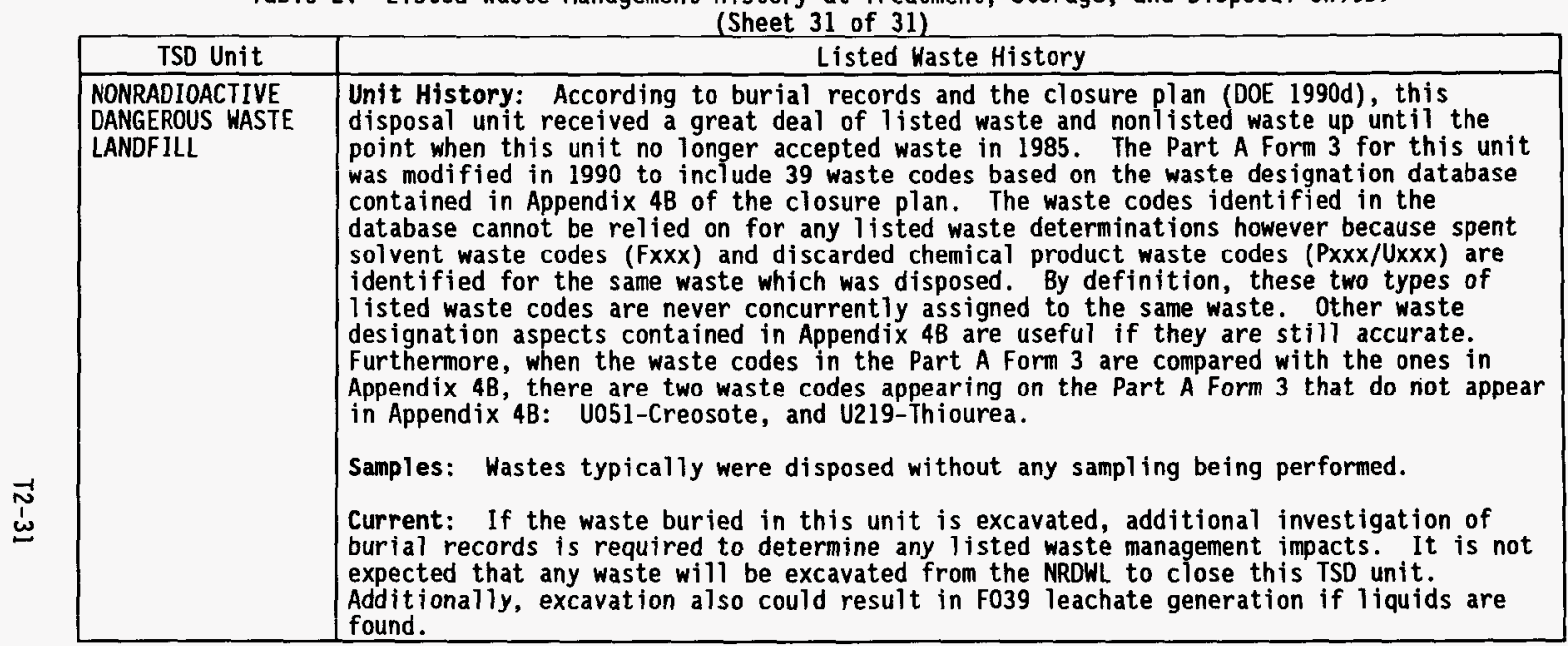


WHC-MR-0517 Rev. 0

This page intentionally left blank. 


\subsection{REFERENCES}

40 CFR 264, "Standards for Owners and Operators of Hazardous Waste Treatment, Storage, and Disposal Facilities," Code of Federal Regulations, as amended.

46 FR 47426, "Hazardous Waste Management System: Identification and Listing of Hazardous Waste: Interim Final Rule," Federal Register, U.S. Environmental Protection Agency, Washington D.C., September 25, 1981.

57 FR 37284, "Identification and Listing of Hazardous Waste; CERCLA Hazardous Substance Designation; Reportable Quantity Adjustment; Coke By-Products Wastes: Final Rule," Federal Register, U.S. Environmental Protection Agency, Washington D.C., August 18, 1992, Section III.B, Response to Comments.

60 FR 6054, "Hazardous Waste Management System; Identification and Listing of Hazardous Waste; Proposed Exclusion," Federal Register, U.S. Environmenta] Protection Agency, Washington D.C., February 1, 1995, 200 Area Effluent Treatment Facility Delisting Petition proposal.

60 FR 31115, "Hazardous Waste Management System; Identification and Listing of Hazardous Waste; Final Exclusion," Federal Register, U.S. Environmental Protection Agency, Washington D.C., June 13, 1995, Granting of 200 Area Effluent Treatment Facility Delisting Petition.

BHI, 1994, letter to J. K. Erickson, RL, from S. D. Liedle, BHI, Review of Waste Designation of Concrete and Soil at 183-H Solar Evaporation Basins, December 21, 1994, Bechtel Hanford, Inc., Rich1and, Washington.

DOE, 1988, Hanford Site Dangerous Waste Part A Permit Application, Revision 13, DOE/RL-88-21, U.S. Department of Energy, Richland Operations Office, Richland, Washington.

DOE, EPA, and Ecology, 1990a, Strategy for Handling of Purgewater at the Hanford Site, Washington, July 1990, U.S. Department of Energy, Richland Operations Office, Richland, Washington.

DOE, 1990b, Remedial Investigation/Feasibility Study Work Plan for the 300-FF-1 Operable unit, Hanford Site, Rich7and, Washington, DOE/RL-88-31, June 1990, U.S. Department of Energy, Richland Operations Office, Richland, Washington.

DOE, 1990C, 216-B-3 Pond System Closure/Post-Closure Plan, Revision 0, DOE/RL-89-28, March 1990, U.S. Department of Energy, Richland Operations Office, Richland, Washington.

DOE, 1990d, Nonradioactive Dangerous Waste Landfill Closure/Post Closure Plan, DOE/RL-90-17, Revision 0, August 1990, U.S. Department of Energy, Richland Operations Office, Richland, Washington.

DOE, 199la, 183-H Solar Evaporation Basins Closure/Post-Closure Plan, Revision 3, DOE/RL-88-04, U.S. Department of Energy, Richland Operations Office, Richland, Washington. 
DOE, 1991b, 616 Nonradioactive Dangerous Waste Storage Facility Dangerous Waste Permit Application, Revision 2, DOE/RL-89-03, September 1991, U.S. Department of Energy, Richland Operations Office, Richland, Washington.

DOE, 1992a, 2727-S Nonradioactive Dangerous Waste Storage Facility Closure Plan, DOE/RL-88-37, Revision 3, U.S. Department of Energy, Richland Operations Office, Richland, Washington.

DOE, 1992b, 300 Area Solvent Evaporator Closure Plan, D0E/RL-88-08, Revision 3B, U.S. Department of Energy, Richland Operations Office, Richland, Washington.

DOE, 1992C, Expedited Response Action Assessment for the 316-5 Trenches, DOE/RL-92-32, Revision 0, U.S. Department of Energy, Richland Operations office, Richi and, Washington.

DOE, 1992d, Hexone Storage and Treatment Facility Closure Plan, DOE/RL-92-40, Revision 0, November 1992, U.S. Department of Energy, Richland Operations office, Richland, Washington.

DOE, 1992e, letter to David B. Jansen, Ecology, from Steven H. Wisness, RL, $T$ Plant Treatment by Generator Request, 92-RPB-32, February 20, 1992, U.S. Department of Energy, Richland Operations Office, Richland, Washington.

DOE, 1992f, 305-B Storage Facility Dangerous Waste Permit Application, DOE/RL-90-01, Revision 2, October 1992, U.S. Department of Energy, Richl and Operations office, Richland, Washington.

DOE, 1993a, 200 Area Effluent Treatment Facility Delisting Petition, DOE/RL-92-72, Revision 1, U.S. Department of Energy, Richland Operations office, Richland, Washington.

DOE, EPA, Ecology, and WHC, 1993b, Meeting minutes, Unit Managers' Meeting 218-E-8 Borrow Pit Demolition Site Closure Plan, Apri1 15, 1993.

DOE, EPA, ECology, and WHC, 1993C, Meeting minutes, Unit Managers' Meeting 218-E-8 Borrow Pit Demolition Site Closure Plan, May 12, 1993.

DOE, EPA, Ecology, and WHC, 1993d, Meeting minutes, Unit Managers' Meeting 218-E-8 Borrow Pit Demolition Site Ciosure Plan, June 10, 1993.

DOE, 1994a, 200 West Area Ashpit Demolition Site Closure Plan, DOE/RL-92-54, Revision 1, U.S. Department of Energy, Richland Operations office, Richiand, Washington.

DOE, 1994b, Hanford Patrol Academy Demolition Sites Closure Plan, DOE/RL-92-39, Revision 1, U.S. Department of Energy, Rich1and Operations office, Richland, Washington.

DOE, 1994C, 216-B-3 Expansion Ponds Closure Plan, D0E/RL-89-28, Revision 2, U.S. Department of Energy, Richland Operations Office, Richland, Washington. 
DOE, 1994d, 218-E-8 Borrow Pit Demolition Site Closure P7an, DOE/RL-92-53, Revision 1, U.S. Department of Energy, Richland Operations Office, Richland, Washington.

DOE, 1995a, The Direct Disposal Team Report: Options for Treating and Disposing of Current and Future Mixed Waste Streams on the Hanford Facility, DOE/RL-93-35, U.S. Department of Energy, Richland Operations Office, Richland, Washington.

DOE, 1995b, 303-K Storage Facility Closure Plan, DOE/RL-90-04, Revision 2A, U.S. Department of Energy, Richland Operations Office, Richland, Washington.

DOE, 1995c, Single-Shell Tank Closure Work Plan, DOE/RL-89-16, Revision A, U.S. Department of Energy, Richland Operations Office, Richland, Washington.

DOE, 1995d, letter to Steve M. Alexander, Ecology and Douglas R. Sherwood, EPA, from James E. Rasmussen, RL and Steven D. Liedle, BHI, Waste Designation of Concrete and Soil at 183-H Solar Evaporation Basins, February 22, 1995, U.S. Department of Energy, Richland Operations Office, Richl and, Washington.

DOE, 1995e, letter to Steve M. Alexander, Ecology, from James E. Rasmussen, RL, and W. T. Dixon, WHC, Proposal to Manage Contaminated Media from the 300 Area Process Trenches (APT), March 14, 1995, U.S. Department of Energy, Richland Operations Office, Richland, Washington.

DOE, 1995f, letter to Shaun McGravey, EPA-HQ, from Thomas K. Teynor, RL, 183-H Basin Waste Equivalent Treatment Petition of Stabilization for the Formic Acid Treatment Standard, October 17, 1995, U.S. Department of Energy, Richland Operations Office, Richland, Washington.

DOE, 1995g, 300 Area Process Trenches Modified Closure/Post Closure Plan, DOE/RL-93-73, Revision 1, U.S. Department of Energy, Richland Operations Office, Richland, Washington.

DOE, 1995h, letter to J.F. Nemec, BHI, from Linda K. MCClain, RL, Contract $D E-A C 06-93 R L 12367$ - Disposed of Scabbled Concrete from the 183-H Basin, October 20, 1995, U.S. Department of Energy, Richland Operations Office, Richland, Washington.

DOE, 1996, 300 Area Waste Acid Treatment, System Closure P7an, DOE/RL-90-11, Revision 1, U.S. Department of Energy, Richland Operations Office, Richland, Washington.

DOE, EPA, Ecology, and WHC, 1995g, Meeting minutes, 200 Area Effluent Treatment Facility (ETF) and Liquid Effluent Retention Facility (LERF) and 242-A Evaporator Unit Manager Meeting, August 31, 1995.

DOE, EPA, Ecology, and WHC, 1995h, Meeting minutes, PUREX Plant/Storage Tunnels Unit Managers Meeting, August 9, 1995.

Ecology, 1994, Hanford Facility RCRA Permit, Dangerous Waste Portion, as amended, Washington State Department of Ecology, Olympia, Washington. 
Ecology, 1995a, letter to James Rasmussen, RL, from Mike Wilson, Ecology, Listed Waste from Hanford Laboratories, March 7, 1995

Ecology, 1995b, letter to James E. Rasmussen, RL, and Robert E. Traister, WHC, from Robert J. Julian, Ecology, Effluent Treatment Facility Delisting Petition, December 7, 1995, Washington State Department of Ecology, Olympia, Washington.

Ecology; 1995c, letter to James Rasmussen, RL, from Steven M. Alexander, Ecology, Conditional Contained-In Determination for the 300 Area Process Trenches, May 15, 1995, Washington State Department of Ecology, 0lympia, Washington.

Ecology, and EPA, 1995d, letter to James E. Rasmussen, RL, and James E. Mecca, $\mathrm{RL}$, from Moses Jaraysi, Ecology, and Dan Duncan, EPA, Response to "Resolution of Permitting and Interim Status Compliance Related Issues Associated with Transition of the Plutonium-Uranium Extraction (PUREX) Facility," dated October 28, 1994, February 21, 1995, Washington State Department of Ecology, 01 ympia, Washington.

Ecology, 1995e, letter to James E. Rasmussen, RL, from Joseph S. Stohr, Ecology, 183-H Solar Evaporation Basin Conditional Contained-in Determination, September 29, 1995, Washington State Department of Ecology, Olympia, Washington.

Ecology, 1996a, letter to James E. Rasmussen, RL, from Ted A. Wooley, Ecology, Conditional Management of 300 Area Process Trench Soils for Waste Code U210, January 16, 1996, Washington State Department of Ecology, Olympia, Washington.

Ecology, 1996b, letter to Kevin Bazze11, RL, from Ted A. Wooley, Ecology, Disposal of Backlog Soil Drums and Boxes Currently stored in Central Waste Complex, February 6, 1996, Washington State Department of Ecology, 0lympia, Washington.

Ecology, 1996c, letter to Kevin Bazze11, RL, from Ted A. Wooley, Ecology, Sampling and Analysis Plan Approval Process for the Backlog Soil Containers in the Central Waste Complex, March 27, 1996, Washington State Department of Ecology, 0lympia, Washington.

Ecology, 1996d, letter to James E. Rasmussen, RL, and A. J. Diliberto, WHC, from Robert J. Julian, Ecology, Hanford Facility Dangerous Waste Permit Application, Form 3 for the Liquid Effluent Retention Facility (LERF), Revision 4, February 21, 1996, Washington State Department of Ecology, 01 ympia, Washington.

Ecology, EPA, and DOE, 1996e, Hanford Federal Facility Agreement and Consent Order, as amended, Washington State Department of Ecology, U.S.

Environmental Protection Agency, and U.S. Department of Energy, Olympia, Washington.

Ecology, 1996f, letter to James E. Rasmussen, RL., from Jeanne Wallace, Ecology, May 13, 1996, pertaining to the closure of two TSD units: Physical/Chemical Treatment Facility and Thermal Treatment Test Facility, Washington State Department of Ecology, 01ympia, Washington. 
EPA, 1989, letter to Howard Wilson, EPA, from Devereaux Barnes, EPA, Classification of Solvent and Commercial Chemical Product Waste Streams, June 28, 1989, RCRA Compendium number 9444.1989(03), U.S. Environmental Protection Agency, Washington, DC.

EPA, 1995, 1etter to Julie K. Erickson, RL, from Betty Wiese, EPA Region 10, Management of Hazardous Debris Contaminated With Listed Waste at the 183-H Solar Evaporation Basins, September 8, 1995, U.S. Environmental Protection Agency, Seattle, Washington.

PNL, 1984a, Laboratory Record Book, BNW50682, Battelle, Pacific Northwest Laboratory, Richland, Washington.

PNL, 1984b, Environmental Monitoring at Hanford for 1984, PNL-5407, Pacific Northwest Laboratory, Richland, Washington.

PNL, 1985a, letter to Barry L. Vedder, UNC, from Khris B. 01sen, PNL, April 2, 1985, B33709, 183-H Analytical Results, Battelle, Pacific Northwest Laboratory, Richland, Washington.

PNL, 1985b, letter to Barry L. Vedder, UNC, from Khris B. 01sen, PNL, Apri1 26, 1985, B32598, 183-H Analytical Results, Battelle, Pacific Northwest Laboratory, Richiland, Washington.

PNL, 1991, letter to Matthew J. Galbraith, WHC, from Bruce A. Prentice, PNL, Final Report of Formate Analysis of 183-H Basin Concrete, November 27, 1991, Battelle, Pacific Northwest Laboratory, Richland, Washington.

Resource Conservation and Recovery Act of 1976, 42 USC 6901 et seq.

RHO, 1984, TSD Facility Annual Dangerous Waste Report 1984, Page 32 of 38, Rockwell Hanford Operations, Richland, Washington.

RHO, 1986a, TSD Facility Annual Dangerous Waste Report 1986 Form 5, Page 21 of 21, Rockwell Hanford Operations, Richland, Washington.

RHO, 1986b, letter to B. J. Carlson, UNC, from J. F. Albaugh, RHO, P7an of Action for Analysis of 183-H Solar Evaporation Basin, R86-0538, Rockwell Hanford Operations, Richland, Washington.

RHO, 1986c, Accidental Discharge of Hydrazine to the PUREX Chemical Sewer, Unusual Occurrence Report 86-36, July 7, 1986, Rockwell Hanford Operations, Richland, Washington.

RHO, 1987, Rockwell Hanford Operations Environmental Surveillance Annual Report Calendar Year 1986, RHO-HS-SR-86-13P, May 1987, Rockwe11 Hanford Operations, Richland, Washington.

Tri-City Herald, 1986, 2 Gallons of Chemicals Spill, July 8, 1986, Tri-City Herald, Kennewick, Washington.

Tri-City Herald, 1989, Operator Error Blamed for Release of Chemica7, August 17, 1989, Tri-City Hera1d, Kennewick, Washington.

UNC, 1981, Perchloroethylene Spill From Transfer Line, Occurrence Report U0-81-1, January 5, 1981, UNC Nuclear Industries, Richland, Washington. 
UNC, 1982, Perchloroethylene Spill into 313 Building Process Sewer, Occurrence Report SO-82-23, November 8, 1982, UNC Nuclear Industries, Richland, Washington.

UNC, 1985a, letter to Distribution, UNC, from Barry. L. Vedder, UNC, Waste Characterization, 183-H Basin No. 1, May 2 1985, UNC Nuclear Industries, Richland, Washington.

UNC, 1985b, Waste Characterization - 183-H Basin No. 1, UNI-3514, August 20, 1985, UNC Nuclear Industries, Richland, Washington.

UNC, 1986, Engineering Plan for the 183-H Solar Evaporation Basin Liquid and Solid Removal/Disposal - Phase I, UNI-4007, June 12, 1986, UNC Nuclear Industries, Richland, Washington.

UNC, 1987a, UNC Environmental Surveillance Report for the 100 Areas --FY1986, UNI-4065, January 12, 1987, UNC Nuclear Industries, Richland, Washington.

UNC, 1987b, letter to T. B. Veneziano, RHO, from D. L. Renberger, UNC, UNC 300 Area Spill Input for 3004(u) Report, March 3, 1987, UNC Nuclear Industries, Richland, Washington.

UNC, 1988, Mix Hydrazine and Morpholine Solutions in 184-N Building, UNI-M-20 Procedure number II-09-23, August 18, 1988, UNC Nuclear Industries, Richland, Washington.

WAC 173-303, "Dangerous Waste Regulations," Washington Administrative Code, as amended.

WHC, 1985, Environmental Protection Manual, MG-99, Section 8.0 Dangerous Waste Control, May 28, 1985, Westinghouse Hanford Company, Richland, Washington.

WHC, 1987a, 300 Area Process Trench Sediment Analysis Report, WHC-SP-0193, Westinghouse Hanford Company, Richland, Washington.

WHC, 1987b, letter to G. J. Bracken, DOE-RL, from H. F. Daugherty, WHC, 183-H Basin Waste Characterization Report, 8753049, Westinghouse Hanford Company, Richland, Washington.

WHC, 1988a, Environmental Investigations and Site Characterization Manual, WHC-CM-7-7, Westinghouse Hanford Company, Richland, Washington.

WHC, 1988b, Westinghouse Hanford Company Environmental Surveillance Annual Report--100 Areas Calendar Year 1987, WHC-EP-0161, Ju1y 1988, Westinghouse Hanford Company, Richland, Washington.

WHC, 1988c, Handle Spills in AMU, P\&O Gallery, and Sample Gallery, PUREX Plant Operation Procedure, PO-020-285 Rev/Mod B-1, May 18, 1988, Westinghouse Hanford Company, Richland, Washington.

WHC, 1988d, Westinghouse Hanford Company Environmental Surveillance Annual Report--200/600 Areas Calendar Year 1987, WHC-EP-0145, April 1988, Westinghouse Hanford Company, Richland, Washington. 
WHC, 1989a, letter to J. R. Hunter, DOE-RL, from H. L. Debban, WHC, Initial Report Unusual Occurrence Report WHC-U0-89-042-100N-03, 8954954, Westinghouse Hanford Company, Richland, Washington.

WHC, 1989b, Chemical Spill Prevention, Control, and Countermeasures P1an --100 Areas, WHC-EP-0460, March 1989, Westinghouse Hanford Company, Richland, Washington.

WHC, 1990a, Westinghouse Hanford Company Environmental Surveill ance Annual Report--100 Areas Calendar Year 1988, WHC-EP-0258, February 1990, Westinghouse Hanford Company, Richland, Washington.

WHC, 1990b, letter to J. R. Hunter, DOE-RL, from H. L. Debban, WHC, Interim Report-Revision No. 1 Unusual Occurrence Report WHC-U0-89-026-100N-01, 9050844, Westinghouse Hanford Company, Richland, Washington.

WHC, 1990C, Fill TK-220/221A or TK-217 with Hydrazine, PUREX P1ant Operation Procedure, P0-120-017 Rev/Mod C-9, October 10, 1990, Westinghouse Hanford Company, Richland, Washington.

WHC, 1990d, 242-A Evaporator Process Condensate Stream Specific Report, WHC-EP-0342 Addendum 15, August 1990, Westinghouse Hanford Company, Richland, Washington.

WHC, 1991, Solid Waste Information and Tracking System (SWITS), March 1991, Westinghouse Hanford Company, Richland, Washington.

WHC, 1992a, Westinghouse Hanford Company Environmental Surveillance Annual Report--100 Areas Calendar Year 1990, WHC-EP-0258-2, May 1992, Westinghouse Hanford Company, Richland, Washington.

WHC, 1992b, 316-5 Process Trenches: Expedited Response Action Sampling Plan, WHC-SD-EN-AP-055, Revision 1, February 1992, Westinghouse Hanford Company, Richland, Washington.

WHC, 1992c, letter to G. T. Berl in et al, WHC, from C. J. Gejer, WHC, Addition of Listed Waste Codes F001 and F002 to Affected Part A Dangerous Waste Permit Applications, March 23, 1995, 81100-92-026, West inghouse Hanford Company, Richland, Washington.

WHC, 1993, Tetter to S. M. Price, WHC, from B. G. Erlandson, WHC, Addition of Listed Waste Code F004 to Affected Dangerous Waste Part A Permit Applications, 88400-93-006, February 18, 1993, Westinghouse Hanford Company, Richland, Washington.

WHC, 1994a, Chain of Custody Records for HPADS Closure, SAF No. 94-390, September 27, 1994, Westinghouse Hanford Company, Richland, Washington.

WHC, 1994b, Chain of Custody Records for 200 West Ash Pit Closure, SAF No. 94-329, July 14, 1994, West inghouse Hanford Company, Richland, Washington.

WHC, 1994c, Chain of Custody Records for 200 West Ash Pit Closure, SAF No. S4-090, December 16, 1994, Westinghouse Hanford Company, Richland, Washington. 
WHC, 1994d, Chain of Custody Records for 218-E-8 Borrow Pit Closure, SAF No. 94-330, July 15, 1994, Westinghouse Hanford Company, Richland, Washington.

WHC, 1994e, Preparation of Mounts for Liquid Scintillation Counting, 222-S Laboratory Procedure, LA-548-111 Rev/Mod A-4, November 28, 1994, Westinghouse Hanford Company, Richland, Washington.

WHC, 1995a, letter to A. J. Diliberto, WHC, from W. T. Dixon, WHC, Listed Waste From Hanford Laboratories, October 23, 1995, Westinghouse Hanford Company, Richland, Washington.

WHC, 1995b, letter to J. D. Wagoner, RL, from A. LaMar Trego, WHC, Listed Waste From Hanford Laboratories, October 23, 1995, 9655488, Westinghouse Hanford Company, Richland, Washington.

WHC, 1995c, letter to W. S. Thompson, BHI, from R. A. Esch, WHC, Results From the $1301 N / 1325 N$ Cribs, November 29, 1995, Westinghouse Hanford Company, Richland, Washington.

WHC, 1996, Process Knowledge Pertaining to Hydrazine and Spent Solvent Listed Waste Sources at the 1301-N Liquid Waste Disposal Facility and the 1325-N Liquid Waste Disposal Facility TSD units, April 10, 1996, Westinghouse Hanford Company, Richland, Washington. 
WHC-MR-0517 Rev. 0

\section{DISTRIBUTION}

Number of copies

ONSITE

1

U.S. Department of Energy, Richland Operations office

DOE-RL Public Reading Room H2-53

7

Bechtel Hanford Incorporated. Environmental Restoration Contractor

J. W. Badden

J. H. Dunkirk

H9-11

R. L. Landon

HO-09

P. J. Mackey

HO- 18

J. E. Rugg

HO- 09

B. L. Vedder

$\times 5-53$

J. P. Zoric

HO-18

$\times 5-57$

5

Pacific Northwest National Laboratory

E. A. Flores

T. J. Lazarski

P7-79

M. J. Riess

P7-79

H. T. Tilden

P7-75

G. T. Thornton

P7-79

P8-45

Westinghouse Hanford Company

D. Alison

R1-51

N. A. Ballantyne

S6-71

B. M. Barnes

T3-04

M. W. Bowman

R1-51

R. C. Bowman

P. J. Davis-Vedder

H6-24

H6-22

M. L. Dexter

W. T. Dixon

R1-51

M. D. Ellefson

H6-21

K. A. Elsethagen

S6-30

R1-51

B. G. Erl andson

R2-36

D. L. Flyckt

L. A. Garner

S6-71

R2-36

C. K. Girres

M. J. Hall

T3-28

T6-12

S. E. Killoy

54-66

G. J. LeBaron

S6-19

B. A. Mayanscik

T3-04

A. G. Miskho (5 copies)

C. H. Mulkey

H6-22

R1-51

F. A. Ruck

H6-23 
WHC-MR-0517 Rev. 0

Number of copies

Westinghouse Hanford Company (cont.)

M. J. Stephenson

H6-20

R. W. Szelmeczka

L6-05

W. E. Toebe

R. D. Pierce

S. M. Price

D. E. Rasmussen

H6-22

T3-04

J. A. Remaize

G. J. Warwick

J. L. Westcott

H6-23

$\mathrm{N} 1-47$

L6-26

T6-12

T3-04

B. D. Williamson

B3-15

Central Files

A3-88

Document Processing Center

A3-94

2 ICF Kaiser Hanford Company

B. J. Dixon

B4-20

C. A. Steward

B4-20 